Brazilian Journal

of Chemical

Engineering

\title{
DEEP EUTECTIC SOLVENTS BASED ON BETAINE AND PROPYLENE GLYCOL AS POTENTIAL DENITRIFICATION AGENTS: A LIQUID-LIQUID EQUILIBRIUM STUDY
}

\author{
Marko Rogošić ${ }^{1}$, Anđela Krišto ${ }^{1}$ and Kristina Zagajski Kučan ${ }^{1 *}$ \\ ${ }^{1}$ University of Zagreb, Faculty of Chemical Engineering and Technology, Zagreb, Croatia. ORCID: 0000-0002-2341-0067; \\ ORCID: 0000-0001-8339-8541; E-mail: kzkucan@fkit.hr - ORCID: 0000-0002-5123-0733
}

(Submitted: January 31, 2019 ; Revised: April 19, 2019 ; Accepted: April 22, 2019)

\begin{abstract}
Two deep eutectic solvents (DES) based on betaine (B) and propylene glycol (PG) in molar ratios of 1:4 and 1:5 were prepared and their physico-chemical properties were determined. Liquid-liquid equilibria at $298.15 \mathrm{~K}$ and atmospheric pressure were determined for 13 quasi-three-component systems with a hydrocarbon ( $n$-hexane, $n$-heptane, $i$-octane or toluene), solute (pyridine or thiophene) and DES (B-PG 1:4 or B-PG 1:5). Experimental phase diagrams and calculated distribution ratios showed that DESs are more suitable for denitrification than for desulfurization. The equilibria in the investigated systems were described by the NRTL and UNIQUAC models, and good agreement with experiments was obtained.

Keywords: Deep eutectic solvents; Liquid-liquid equilibrium; Fuel purification; NRTL; UNIQUAC.
\end{abstract}

\section{INTRODUCTION}

In accordance with the principles of "green chemistry", it is generally attempted to reduce the consumption of hazardous substances or to completely eliminate them from chemical processes. This refers to raw materials, reagents, solvents, products and byproducts, but also includes the use of renewable raw materials and energy sources for production processes. Petroleum-based fuels, both as raw materials and as products, fall into the class of non-renewable sources of energy, making them "non-green" in the sense of the above principles. Their "greening" can be achieved by replacing a part of the fuel by one produced from renewable sources and by reducing the harmful emissions of sulfur and carbon oxides generated by fuel combustion. Extraction as an operation for removal of sulfur and nitrogen-containing compounds from hydrocarbon mixtures is, in this sense, certainly "greener" than, e.g., hydrodesulfurization, HDS, and hydrodenitrification, HDN. The latter are the high- energy and costly processes which are today the first choice in industry. The extraction is "greener" than eventual distillation separation as well, as it usually occurs at lower temperatures. Nevertheless, the extraction agent has to be as "green" as possible, too. Classical organic solvents are easily volatile and mostly toxic and are therefore clearly "non-green". Ionic liquids, ILs, are "green" with respect to their low volatility (Plechkova and Seddon, 2008) and regeneration potential (Zhang et al., 2004), but are toxic to microorganisms after releasing into the environment and are also made up of toxic raw materials and semiproducts (Zhao et al., 2007). Deep eutectic solvents, DESs, are essentially much "greener" than ILs in this respect.

DESs are not well-defined chemical species but are, in most cases, two-component mixtures comprising hydrogen bond donor and acceptor moieties, HBD and HBA, respectively, in a molar ratio defined by the mixing itself. The DES components are completely miscible, and miscibility is often promoted by the

\footnotetext{
* Corresponding author: Kristina Zagajski Kučan - E-mail: kzkucan@fkit.hr
} 
presence of hydrogen bonding, which is why they behave non-ideally in the liquid phase. DESs can be prepared from biodegradable components, using simpler methods than is the case with ILs, and they are generally non-toxic and cheaper for synthesis.

The properties that are very important for the application of DESs in extraction are: high capacity for dissolving different types of substances, chemical and thermal stability, low vapour pressure, and non-flammability (Morrison et al., 2009). They are proven to be suitable for regeneration (Gano et al., 2015). DESs interact with different components via hydrogen bonding and van der Waals forces, but also by electrostatic interactions, enabling excellent mixing of DES and polar substances (Li et al., 2013). The properties of DESs can be modified easily by substituting the hydrogen bond donor or acceptor component by some other chemical moiety, or simply by changing their molar ratio. This broadens the potential for application of DESs in various fields (Smith, 2014).

DESs explored so far for extracting sulfur and nitrogen-containing compounds from hydrocarbon solvents are composed mostly of a series of amides and carboxylic acids in combination with quaternary ammonium salts, as well as of alcohols, urea, sugars, or organic acids mixed with choline chloride. In a recent report, DESs based on choline chloride were used for denitrification (Ali et al., 2016). The best results in extraction of nitrogen compounds were achieved by DES consisting of choline chloride and phenylacetic acid in a molar ratio of $1: 2$. This system removes both basic (pyridine, quinoline) and non-basic nitrogen compounds (carbazole, indole, pyrrole). At $308.15 \mathrm{~K}$, 99.2\% pyridine extraction yield and $98.2 \%$ carbazole extraction yield were achieved at a solvent to fuel ratio of $1: 1$, which is a very good result compared to conventional solvents. Abbott et al. (2007) used DES consisting of quaternary ammonium salts and glycerol in the component ratio of 1:2 to extract successfully $(>99 \%)$ sulfur compounds from soybean-oil-based biodiesel in a multistage process.

Real hydrocarbon fuels originating both from nonrenewable and renewable sources are complex mixtures and it is therefore often simpler and easier to observe trends by studying model fuels. Thus desulfurization
(Li et al., 2013; Li et al., 2016; Gano et al., 2015), denitrification (Ali et al., 2016; Hizaddin et al., 2016) and dearomatization processes (Sander et al., 2016) were investigated using DESs and model fuels. An even simpler approach is possible by the experimental study of ternary liquid-liquid equilibria (LLE) in the systems based on DES as a quasi-component. Thereby, NRTL (Hizaddin et al., 2016; Sander et al., 2016; Rogošić and Zagajski Kučan, 2018), UNIQUAC (Rogošić and Zagajski Kučan, 2018) and COSMO-RS (Hizaddin et al., 2016; Mulyono et al., 2014) activity coefficient models were used for the thermodynamic description of phase equilibria in quasi-three-component systems containing DESs.

In this paper, LLE are studied in quasi-threecomponent systems. The key component (pyridine or thiophene) is distributed between a hydrocarbon - either aliphatic ( $n$-hexane, $n$-heptane, $i$-octane) or aromatic (toluene) - and a DES based on betaine and propylene glycol. The quantities important for modeling extraction processes - selectivity and distribution ratio - are also discussed. LLE are described by NRTL or UNIQUAC. In addition, the physico-chemical properties important for the application of the explored DESs are determined as well. The literature review confirms that this is the first study dealing with liquidliquid equilibrium data in the quasi-three-component systems composed of betaine and propylene glycol, hydrocarbons and pyridine or thiophene, with the purpose of contributing data for the development of environmentally friendly fuel purification methods.

\section{EXPERIMENTAL PART}

\section{Chemicals}

The chemicals used are listed in Table 1. No further purification of the chemicals was performed.

\section{Preparation and characterization of DESs}

DES components, betaine and propylene glycol, were weighed into a flask in molar ratios of 1:4 and 1:5. These molar ratios were chosen based on our previous research (Zagajski Kučan et al., 2018) where a molar ratio of 1:3.5 was studied. In an attempt to find DES with the best properties for maximum removal of pyridine and thiophene from the organic mixture,

Table 1. Chemicals.

\begin{tabular}{lclcc}
\hline \multicolumn{1}{c}{ Chemical } & CAS number & \multicolumn{1}{c}{ Manufacturer (Town, Country) } & Purity / wt. \% & Molar mass / g mol \\
\hline$n$-hexane & $110-54-3$ & Carlo Erba Reagents (Val de Reuil, France) & $>98.5$ & 86.18 \\
$n$-heptane & $142-82-5$ & Carlo Erba Reagents (Val de Reuil, France) & 99 & 100.21 \\
$i$-octane & $540-84-1$ & Kemika (Zagreb, Croatia) & $>99.5$ & 114.23 \\
toluene & $108-88-3$ & Lach:ner (Neratovice, Czech Republic) & $>99.3$ & 92.14 \\
thiophene & $110-02-1$ & Acros Organics (Geel, Belgium) & $>99$ & 84.14 \\
pyridine & $110-86-1$ & Carlo Erba Reagents (Val de Reuil, France) & $>99$ & 79.10 \\
betaine & $107-43-7$ & Acros Organics (Geel, Belgium) & 98 & 117.15 \\
propylene glycol & $57-55-6$ & Acros Organics (Geel, Belgium) & 99 & 76.10 \\
\hline
\end{tabular}


the optimal composition, i.e., the optimal molar ratio of HBA: HBD is to be determined first. Thereby, the main constraint is the requirement that DES has to be liquid at the investigated extraction temperature. The mixtures were then heated to $353.15 \mathrm{~K}$ and stirred on a magnetic stirrer for 30 minutes at atmospheric pressure. The end of the preparation was visible when a colorless and homogeneous liquid was obtained. The nominal molar masses of the DESs thus formed were 421.55 and $497.65 \mathrm{~g} \mathrm{~mol}^{-1}$, respectively.

The physico-chemical properties important for the application were determined for both DESs prepared. All the properties were determined at atmospheric pressure and at temperatures of $288.15 \mathrm{~K}, 298.15 \mathrm{~K}$, $308.15 \mathrm{~K}, 318.15 \mathrm{~K}$ and $328.15 \mathrm{~K}$, except for the set of thermal properties, which were determined at $298.15 \mathrm{~K}$ only. The temperature of $288.15 \mathrm{~K}$ was achieved with the use of an ice bath and for other temperatures an electrically heated water bath was used. The instruments used are listed in Table 2.

\section{Determination of tie lines}

In order to obtain an overview of the equilibrium behaviour in the 13 investigated three-component systems, mixtures of one of the hydrocarbons ( $n$-hexane, $n$-heptane, $i$-octane or toluene), pyridine or thiophene and, finally, one of the DESs (B-PG 1:4 or B-PG 1:5) of different compositions were prepared. All the compositions fell within the region in which these unstable mixtures separated into two liquid phases only. The exact compositions of the unstable mixtures prepared were calculated from the masses of the individual components as weighed on an analytical scale. The two-phase systems thus formed were mixed in a thermostated vessel equipped with a magnetic stirrer for $24 \mathrm{~h}$ at $298.15 \mathrm{~K}$ at $200 \mathrm{rpm}$ and at atmospheric pressure. After switching off the stirring, the samples were left for $48 \mathrm{~h}$ to separate into equilibrium phases.

Preliminary experiments have shown that there was practically no DES in the raffinate phase, i.e., one was dealing with two-component mixtures of hydrocarbons with pyridine or toluene, respectively.
Therefore, the compositions of the raffinate phase can be determined from the measured refractive indices, which can be converted into component mass fractions by the previously prepared calibration curves. The refractive indices, both in preparing the calibration curves and in determining tie lines, were measured at 298.15 K using the Abbe refractometer (model RL-3, Poland, precision $\pm 0.0001 n_{\mathrm{D}}$ ).

Preliminary experiments have also shown that hydrocarbons were practically insoluble in the extract phase. The composition of the extract phases was calculated using the material balance, i.e., from the known overall composition and the refractometrically determined composition of the raffinate phase.

\section{RESULTS AND DISCUSSION}

\section{Physico-chemical properties}

The results for thermal properties at $298.15 \mathrm{~K}$ are shown in Table 3; a higher thermal conductivity, $\lambda$, and temperature diffusivity, $a$, was found for B-PG 1:5. The heat capacity of the liquid at constant pressure, $c$, was higher for B-PG 1:4. The results of measurements of other properties are shown in Table 4.

Various empirical expressions can be used to describe the temperature dependence of the measured properties, as is commonly done in the literature (Jibril et al., 2014). Thus, in the investigated temperature range the density decreases linearly with the temperature, with high values of the regression coefficient. The dynamic viscosity of DES diminishes with the temperature, following a fractional rational function of the third order, also with high values of the regression coefficient. Dynamic viscosity values are somewhat higher in B-PG 1:4 than in B-PG 1:5. Specific conductance rises with the temperature, following an exponential law. The refractive index diminishes upon increasing the temperature, approximately linearly in the explored temperature range. Higher values were observed for B-PG 1:4 than for B-PB 1:5.

Applied empirical correlations are presented in Table 5 together with values of parameters and regression coefficients.

Table 2. Determination of physico-chemical properties of DESs.

\begin{tabular}{llc}
\hline \multicolumn{1}{c}{ Property } & \multicolumn{1}{c}{ Measuring device } & Temperature (K) \\
\hline Specific conductance & Schott Instruments Lab 960 conductivity meter & $288.15-328.15$ \\
Refractive index & Abbe refractometer, model RL-3, Poland & $288.15-328.15$ \\
Density & Anton Paar Density Meter DMA 4500 M & $288.15-328.15$ \\
Thermal properties & Thermal conductometer, Linseis Transient Hot Bridge 1 & 298.15 \\
Dynamic viscosity & Brookfield DV-III Ultra V 6.0 Programmable Rheometer; & $288.15-328.15$ \\
\hline
\end{tabular}

Table 3. Temperature diffusivity, $a$, thermal conductivity, $\lambda$, and heat capacity, $c_{p}$, of deep eutectic solvents. The measurements were done at atmospheric pressure.

\begin{tabular}{ccccc}
\hline DES & $\boldsymbol{T} / \mathbf{K}$ & $\boldsymbol{a} / \mathbf{m m}^{\mathbf{2}} \mathbf{s}^{\mathbf{1}}$ & $\lambda / \mathbf{W}(\mathbf{m ~ K})^{-\mathbf{1}}$ & $\boldsymbol{c}_{p} / \mathbf{J}(\mathbf{g ~ K})^{-\mathbf{1}}$ \\
\hline B-PG 1:4 & $298.75 \pm 0.1$ & 0.1057 & 0.2028 & 1.8067 \\
B-PG 1:5 & $298.45 \pm 0.1$ & 0.1370 & 0.2050 & 1.4000 \\
\hline
\end{tabular}


Table 4. Density, $\rho$; dynamic viscosity, $\mu$; specific conductance, $\sigma$; refractive index, $n_{\mathrm{D}}$; as functions of temperature for the investigated deep eutectic solvents. The measurements were done at atmospheric pressure.

\begin{tabular}{ccccccc}
\hline \multirow{2}{*}{ Property } & \multirow{2}{*}{ DES } & \multicolumn{5}{c}{$\boldsymbol{T} / \mathbf{K}$} \\
\cline { 3 - 7 } & & $\mathbf{2 8 8 . 1 5} \pm \mathbf{0 . 1}$ & $\mathbf{2 9 8 . 1 5} \pm \mathbf{0 . 1}$ & $\mathbf{3 0 8 . 1 5} \pm \mathbf{0 . 1}$ & $\mathbf{3 1 8 . 1 5} \pm \mathbf{0 . 1}$ & $\mathbf{3 2 8 . 1 5} \pm \mathbf{0 . 1}$ \\
\hline \multirow{2}{*}{$\rho / \mathrm{kg} \mathrm{m}^{-3}$} & B-PG 1:4 & 1075.21 & 1069.33 & 1062.61 & 1055.96 & 1048.46 \\
& B-PG 1:5 & 1073.24 & 1066.56 & 1059.8 & 1053.08 & 1046.31 \\
$\mu / \mathrm{Pa} \mathrm{s}$ & B-PG 1:4 & 0.2454 & 0.1184 & 0.0616 & 0.0347 & 0.0218 \\
& B-PG 1:5 & 0.2157 & 0.0924 & 0.0514 & 0.03 & 0.0187 \\
$\sigma / \mu \mathrm{S} \mathrm{cm}^{-1}$ & B-PG 1:4 & 3.1 & 4.5 & 7.2 & 11.9 & 19.5 \\
& B-PG 1:5 & 0.3 & 0.5 & 0.7 & 1.1 & 1.8 \\
$n_{\mathrm{D}}$ & B-PG 1:4 & 1.4509 & 1.4498 & 1.4468 & 1.4449 & 1.4422 \\
& B-PG 1:5 & 1.4492 & 1.4470 & 1.4443 & 1.4425 & 1.4412 \\
\hline
\end{tabular}

Table 5. Correlations of density, $\rho$; dynamic viscosity, $\mu$; specific conductance, $\sigma$; refractive index, $n_{\mathrm{D}}$; vs. temperature for investigated deep eutectic solvents.

\begin{tabular}{|c|c|c|c|c|}
\hline$y$-property & $x$-property & DES & Correlation & $\begin{array}{c}\text { Regression coefficient, } \\
R^{2}\end{array}$ \\
\hline \multirow{2}{*}{$\rho / \mathrm{kg} \mathrm{m}^{-3}$} & \multirow{2}{*}{$T / \mathrm{K}$} & B-PG 1:4 & $y=1268.4-0.668 x$ & 0.9977 \\
\hline & & B-PG 1:5 & $y=1267.3-0.673 x$ & 0.9999 \\
\hline \multirow{2}{*}{$\mu / \mathrm{Pa} \mathrm{s}$} & \multirow{2}{*}{$T / \mathrm{K}$} & B-PG 1:4 & $y=-93.42+9.027 \times 10^{4} / x-2.911 \times 10^{7} / x^{2}+3.134 \times 10^{9} / x^{3}$ & 0.9995 \\
\hline & & B-PG 1:5 & $y=-132.8+1.265 \times 10^{5} / x-4.019 \times 10^{7} / x^{2}+4.260 \times 10^{9} / x^{3}$ & 0.9963 \\
\hline \multirow{2}{*}{$\sigma / \mu \mathrm{S} \mathrm{cm}^{-1}$} & \multirow{2}{*}{$T / \mathrm{K}$} & B-PG 1:4 & $y=2.395 \times 10^{-6} \exp (0.0485 x)$ & 0.9988 \\
\hline & & B-PG 1:5 & $y=5.179 \times 10^{-7} \exp (0.0459 x)$ & 0.9950 \\
\hline \multirow{2}{*}{$n_{\mathrm{D}}$} & \multirow{2}{*}{$T / \mathrm{K}$} & B-PG 1:4 & $y=1.516-2.230 \times 10^{-4} x$ & 0.979 \\
\hline & & B-PG 1:5 & $y=1.508-2.058 \times 10^{-4} x$ & 0.977 \\
\hline
\end{tabular}

\section{Tie lines}

For the experimental determination of equilibrium, 13 hydrocarbon (1) - pyridine / thiophene (2) - DES (3) systems were investigated in the composition region where the systems separated into two stable phases in equilibrium. This corresponds to the range of values of total mass fraction of pyridine or thiophene of approximately $w_{2} \leq 0.6$, depending on the system. Systems with similar phase diagrams have already been described in the literature (Sander et al., 2016; Mulyono et al., 2014; Hizaddin et al., 2015; Kareem et al., 2013;
Kareem et al., 2012). The results are shown in the form of triangular diagrams, Figures 1 - 13, and in Table 6.

\section{Selectivity and distribution ratio}

The parameters commonly used to evaluate the suitability of a particular solvent for the separation of pyridine or thiophene from a hydrocarbon are discussed here. Thus, selectivity is defined by the expression:

$$
\mathrm{S}=\frac{\mathrm{w}_{2}^{\mathrm{E}} \mathrm{w}_{1}^{\mathrm{R}}}{\mathrm{w}_{2}^{\mathrm{R}} \mathrm{w}_{1}^{\mathrm{E}}}
$$

Table 6. Experimental and model tie lines in quasi-three-component systems, at $298.15 \mathrm{~K}$ and at atmospheric pressure.

\begin{tabular}{|c|c|c|c|c|c|c|c|c|c|c|c|c|c|}
\hline \multicolumn{14}{|c|}{ n-hexane (1) - pyridine (2) - B-PG 1:4 (3) } \\
\hline \multicolumn{6}{|c|}{ experiment } & \multicolumn{4}{|c|}{ NRTL } & \multicolumn{4}{|c|}{ UNIQUAC } \\
\hline \multicolumn{2}{|c|}{ feed } & \multicolumn{2}{|c|}{ raffinate } & \multicolumn{2}{|c|}{ extract } & \multicolumn{2}{|c|}{ raffinate } & \multicolumn{2}{|c|}{ extract } & \multicolumn{2}{|c|}{ raffinate } & \multicolumn{2}{|c|}{ extract } \\
\hline$w_{2}$ & $w_{3}$ & $w_{2}$ & $w_{3}$ & $w_{2}$ & $w_{3}$ & $w_{2}$ & $w_{3}$ & $w_{2}$ & $w_{3}$ & $w_{2}$ & $w_{3}$ & $w_{2}$ & $w_{3}$ \\
\hline 0.1024 & 0.4257 & 0.038 & 0.000 & 0.165 & 0.835 & 0.0399 & 0.0000 & 0.1640 & 0.8359 & 0.0365 & 0.0000 & 0.1650 & 0.8350 \\
\hline 0.2163 & 0.3480 & 0.097 & 0.000 & 0.327 & 0.673 & 0.0931 & 0.0000 & 0.3289 & 0.6710 & 0.0944 & 0.0000 & 0.3284 & 0.6716 \\
\hline 0.3096 & 0.3189 & 0.128 & 0.000 & 0.444 & 0.556 & 0.1314 & 0.0000 & 0.4431 & 0.5568 & 0.1348 & 0.0000 & 0.4418 & 0.5582 \\
\hline 0.4101 & 0.2897 & 0.168 & 0.000 & 0.547 & 0.453 & 0.1658 & 0.0000 & 0.5475 & 0.4524 & 0.1677 & 0.0000 & 0.5468 & 0.4532 \\
\hline 0.5160 & 0.2372 & 0.199 & 0.000 & 0.657 & 0.343 & 0.1999 & 0.0000 & 0.6569 & 0.3430 & 0.1963 & 0.0000 & 0.6581 & 0.3419 \\
\hline \multicolumn{14}{|c|}{ n-heptane (1) - pyridine (2) - B-PG 1:4 (3) } \\
\hline \multicolumn{6}{|c|}{ experiment } & \multicolumn{4}{|c|}{ NRTL } & \multicolumn{4}{|c|}{ UNIQUAC } \\
\hline \multicolumn{2}{|c|}{ feed } & \multicolumn{2}{|c|}{ raffinate } & \multicolumn{2}{|c|}{ extract } & \multicolumn{2}{|c|}{ raffinate } & \multicolumn{2}{|c|}{ extract } & \multicolumn{2}{|c|}{ raffinate } & \multicolumn{2}{|c|}{ extract } \\
\hline$w_{2}$ & $w_{3}$ & $w_{2}$ & $w_{3}$ & $w_{2}$ & $w_{3}$ & $w_{2}$ & $w_{3}$ & $w_{2}$ & $w_{3}$ & $w_{2}$ & $w_{3}$ & $w_{2}$ & $w_{3}$ \\
\hline 0.1046 & 0.4365 & 0.250 & 0.000 & 0.668 & 0.332 & 0.2446 & 0.0000 & 0.6698 & 0.3302 & 0.2404 & 0.0005 & 0.6715 & 0.3284 \\
\hline 0.2094 & 0.3618 & 0.072 & 0.000 & 0.137 & 0.863 & 0.0623 & 0.0000 & 0.1400 & 0.8600 & 0.0394 & 0.0288 & 0.1487 & 0.8513 \\
\hline 0.3078 & 0.3307 & 0.098 & 0.000 & 0.310 & 0.690 & 0.0943 & 0.0000 & 0.3118 & 0.6882 & 0.0873 & 0.0139 & 0.3165 & 0.6835 \\
\hline 0.4209 & 0.2752 & 0.131 & 0.000 & 0.434 & 0.566 & 0.1271 & 0.0000 & 0.4354 & 0.5646 & 0.1282 & 0.0066 & 0.4365 & 0.5635 \\
\hline 0.5208 & 0.2154 & 0.164 & 0.000 & 0.568 & 0.432 & 0.1774 & 0.0000 & 0.5621 & 0.4379 & 0.1810 & 0.0022 & 0.5613 & 0.4387 \\
\hline
\end{tabular}


Table 6. Continuation

\begin{tabular}{|c|c|c|c|c|c|c|c|c|c|c|c|c|c|}
\hline \multicolumn{14}{|c|}{$i$-octane (1) - pyridine (2) - B-PG 1:4 (3) } \\
\hline \multicolumn{6}{|c|}{ experiment } & \multicolumn{4}{|c|}{ NRTL } & \multicolumn{4}{|c|}{ UNIQUAC } \\
\hline \multicolumn{2}{|c|}{ feed } & \multicolumn{2}{|c|}{ raffinate } & \multicolumn{2}{|c|}{ extract } & \multicolumn{2}{|c|}{ raffinate } & \multicolumn{2}{|c|}{ extract } & \multicolumn{2}{|c|}{ raffinate } & \multicolumn{2}{|c|}{ extract } \\
\hline$w_{2}$ & $w_{3}$ & $w_{2}$ & $w_{3}$ & $w_{2}$ & $w_{3}$ & $w_{2}$ & $w_{3}$ & $w_{2}$ & $w_{3}$ & $w_{2}$ & $w_{3}$ & $w_{2}$ & $w_{3}$ \\
\hline 0.1044 & 0.4388 & 0.058 & 0.000 & 0.148 & 0.852 & 0.0519 & 0.0000 & 0.1505 & 0.8495 & 0.0333 & 0.0229 & 0.1588 & 0.8412 \\
\hline 0.2170 & 0.3512 & 0.084 & 0.000 & 0.336 & 0.664 & 0.0814 & 0.0000 & 0.3369 & 0.6631 & 0.0765 & 0.0092 & 0.3409 & 0.6591 \\
\hline 0.3053 & 0.3412 & 0.102 & 0.000 & 0.437 & 0.563 & 0.1034 & 0.0000 & 0.4366 & 0.5634 & 0.1047 & 0.0047 & 0.4373 & 0.5627 \\
\hline 0.4096 & 0.2745 & 0.142 & 0.000 & 0.566 & 0.434 & 0.1452 & 0.0000 & 0.5640 & 0.4360 & 0.1508 & 0.0014 & 0.5620 & 0.4380 \\
\hline 0.5232 & 0.2169 & 0.214 & 0.000 & 0.676 & 0.324 & 0.2130 & 0.0000 & 0.6763 & 0.3237 & 0.2080 & 0.0003 & 0.6784 & 0.3216 \\
\hline
\end{tabular}

n-hexane (1) - thiophene (2) - B-PG 1:4 (3)

\begin{tabular}{|c|c|c|c|c|c|c|c|c|c|c|c|c|c|}
\hline \multicolumn{6}{|c|}{ experiment } & \multicolumn{4}{|c|}{ NRTL } & \multicolumn{4}{|c|}{ UNIQUAC } \\
\hline \multicolumn{2}{|c|}{ feed } & \multicolumn{2}{|c|}{ raffinate } & \multicolumn{2}{|c|}{ extract } & \multicolumn{2}{|c|}{ raffinate } & \multicolumn{2}{|c|}{ extract } & \multicolumn{2}{|c|}{ raffinate } & \multicolumn{2}{|c|}{ extract } \\
\hline$w_{2}$ & $w_{3}$ & $w_{2}$ & $w_{3}$ & $w_{2}$ & $w_{3}$ & $w_{2}$ & $w_{3}$ & $w_{2}$ & $w_{3}$ & $w_{2}$ & $w_{3}$ & $w_{2}$ & $w_{3}$ \\
\hline 0.1036 & 0.5633 & 0.225 & 0.000 & 0.069 & 0.931 & 0.2183 & 0.0001 & 0.0713 & 0.9287 & 0.2206 & 0.0000 & 0.0706 & 0.9294 \\
\hline 0.2121 & 0.3720 & 0.295 & 0.000 & 0.093 & 0.907 & 0.3057 & 0.0002 & 0.0888 & 0.9112 & 0.2998 & 0.0000 & 0.0911 & 0.9090 \\
\hline 0.3119 & 0.3263 & 0.430 & 0.000 & 0.107 & 0.893 & 0.4249 & 0.0003 & 0.1092 & 0.8908 & 0.4289 & 0.0000 & 0.1075 & 0.8926 \\
\hline \multicolumn{14}{|c|}{ n-heptane (1) - thiophene (2) - B-PG 1:4 (3) } \\
\hline \multicolumn{6}{|c|}{ experiment } & \multicolumn{4}{|c|}{ NRTL } & \multicolumn{4}{|c|}{ UNIQUAC } \\
\hline \multicolumn{2}{|c|}{ feed } & \multicolumn{2}{|c|}{ raffinate } & \multicolumn{2}{|c|}{ extract } & \multicolumn{2}{|c|}{ raffinate } & ext & act & raff & late & ext & act \\
\hline$w_{2}$ & $w_{3}$ & $w_{2}$ & $w_{3}$ & $w_{2}$ & $w_{3}$ & $w_{2}$ & $w_{3}$ & $w_{2}$ & $w_{3}$ & $w_{2}$ & $w_{3}$ & $w_{2}$ & $w_{3}$ \\
\hline 0.0665 & 0.4195 & 0.110 & 0.000 & 0.006 & 0.994 & 0.1181 & 0.0000 & 0.0044 & 0.9956 & 0.0821 & 0.0000 & 0.0137 & 0.9863 \\
\hline 0.1042 & 0.4229 & 0.154 & 0.000 & 0.041 & 0.959 & 0.1543 & 0.0000 & 0.0406 & 0.9594 & 0.1611 & 0.0000 & 0.0385 & 0.9615 \\
\hline 0.2148 & 0.3533 & 0.277 & 0.000 & 0.123 & 0.877 & 0.2954 & 0.0000 & 0.1150 & 0.8850 & 0.2967 & 0.0000 & 0.1147 & 0.8847 \\
\hline 0.3369 & 0.2752 & 0.417 & 0.000 & 0.176 & 0.824 & 0.4167 & 0.0000 & 0.1768 & 0.8232 & 0.3999 & 0.0005 & 0.1864 & 0.8106 \\
\hline & & & & & -octane & (1) - thio & hene (2) & B-PG 1: & (3) & & & & \\
\hline & & experi & nent & & & & & & & & UNI & JAC & \\
\hline & & raffi & nate & ext & & raft & ate & ext & act & raff & ate & ext & act \\
\hline$w_{2}$ & $w_{3}$ & $w_{2}$ & $w_{3}$ & $w_{2}$ & $w_{3}$ & $w_{2}$ & $w_{3}$ & $w_{2}$ & $w_{3}$ & $w_{2}$ & $w_{3}$ & $w_{2}$ & $w_{3}$ \\
\hline 0.0605 & 0.4460 & 0.157 & 0.000 & 0.046 & 0.954 & 0.1538 & 0.0002 & 0.0468 & 0.9532 & 0.1586 & 0.0000 & 0.0452 & 0.9549 \\
\hline 0.1071 & 0.4265 & 0.299 & 0.000 & 0.081 & 0.919 & 0.3040 & 0.0005 & 0.0788 & 0.9212 & 0.2972 & 0.0000 & 0.0817 & 0.9183 \\
\hline 0.2112 & 0.3690 & 0.439 & 0.000 & 0.108 & 0.892 & 0.4486 & 0.0007 & 0.1023 & 0.8977 & 0.4394 & 0.0000 & 0.1075 & 0.8925 \\
\hline 0.3198 & 0.3211 & 0.551 & 0.000 & 0.112 & 0.888 & 0.5399 & 0.0009 & 0.1189 & 0.8811 & 0.5507 & 0.0000 & 0.1124 & 0.8876 \\
\hline & & & & & t-hexar & (1) - py & dine (2) & B-PG 1: & & & & & \\
\hline & & experi & nent & & & & & & & & UNI & $\mathrm{UAC}$ & \\
\hline & & raff & nate & ext & & raff & ate & ext & act & raff & late & ext & act \\
\hline$w_{2}$ & $w_{3}$ & $w_{2}$ & $w_{3}$ & $w_{2}$ & $w_{3}$ & $w_{2}$ & $w_{3}$ & $w_{2}$ & $w_{3}$ & $w_{2}$ & $w_{3}$ & $w_{2}$ & $w_{3}$ \\
\hline 0.1076 & 0.4188 & 0.060 & 0.000 & 0.156 & 0.844 & 0.0606 & 0.0000 & 0.1558 & 0.8439 & 0.0387 & 0.0227 & 0.1627 & 0.8372 \\
\hline 0.2059 & 0.3834 & 0.093 & 0.000 & 0.299 & 0.701 & 0.0903 & 0.0000 & 0.3002 & 0.6996 & 0.0773 & 0.0107 & 57 & 0.6943 \\
\hline 0.3312 & 0.2932 & 0.132 & 0.000 & 0.483 & 0.517 & 0.1374 & 0.0000 & 0.4811 & 0.5188 & 0.1404 & 0.0026 & 0.4807 & 0.5193 \\
\hline 0.4213 & 0.2735 & 0.174 & 0.000 & 0.566 & 0.434 & 0.1719 & 0.0000 & 0.5670 & 0.4330 & 0.1813 & 0.0010 & 0.5639 & 0.4361 \\
\hline 0.5445 & 0.2033 & 0.271 & 0.000 & 0.689 & 0.311 & 0.2709 & 0.0000 & 0.6892 & 0.3108 & 0.2654 & 0.0001 & 0.6911 & 0.3089 \\
\hline & & & & & -hepta & (1) - py & idine (2) & B-PG 1: & (3) & & & & \\
\hline & & experi & nent & & & & & TL & & & UNI & UAC & \\
\hline & & raff & late & ext & & raff & ate & & act & raff & late & ext & act \\
\hline$w_{2}$ & $w_{3}$ & $w_{2}$ & $w_{3}$ & $w_{2}$ & $w_{3}$ & $w_{2}$ & $w_{3}$ & $w_{2}$ & $w_{3}$ & $w_{2}$ & $w_{3}$ & $w_{2}$ & $w_{3}$ \\
\hline 0.1012 & 0.4379 & 0.070 & 0.000 & 0.132 & 0.868 & 0.0637 & 0.0000 & 0.1340 & 0.8660 & 0.0750 & 0.0000 & 0.1304 & 0.8695 \\
\hline 0.1994 & 0.3942 & 0.106 & 0.000 & 0.278 & 0.722 & 0.0939 & 0.0000 & 0.2820 & 0.7180 & 0.0923 & 0.0000 & 0.2825 & 0.7171 \\
\hline 0.3039 & 0.3384 & 0.134 & 0.000 & 0.423 & 0.577 & 0.1335 & 0.0000 & 0.4237 & 0.5763 & 0.1313 & 0.0000 & 0.4236 & 0.5734 \\
\hline 0.4164 & 0.2795 & 0.172 & 0.000 & 0.558 & 0.442 & 0.1866 & 0.0000 & 0.5521 & 0.4479 & 0.1835 & 0.0000 & 0.5487 & 0.4408 \\
\hline 0.5134 & 0.2353 & 0.252 & 0.000 & 0.646 & 0.354 & 0.2443 & 0.0000 & 0.6486 & 0.3514 & 0.2475 & 0.0000 & 0.6352 & 0.3418 \\
\hline & & & & & $i$-octan & (1) - pyr & line (2) - & 3-PG 1:5 & & & & & \\
\hline & & experi & nent & & & & & & & & UNIC & UAC & \\
\hline & & raff & nate & ext & act & raff & ate & ext & act & raff & ate & ext & act \\
\hline$w_{2}$ & $w_{3}$ & $w_{2}$ & $w_{3}$ & $w_{2}$ & $w_{3}$ & $w_{2}$ & $w_{3}$ & $w_{2}$ & $w_{3}$ & $w_{2}$ & $w_{3}$ & $w_{2}$ & $w_{3}$ \\
\hline 0.1011 & 0.4497 & 0.053 & 0.000 & 0.145 & 0.855 & 0.0504 & 0.0000 & 0.1456 & 0.8544 & 0.0498 & 0.0000 & 0.1459 & 0.8542 \\
\hline 0.2017 & 0.3931 & 0.071 & 0.000 & 0.303 & 0.697 & 0.0752 & 0.0000 & 0.3009 & 0.6991 & 0.0736 & 0.0000 & 0.3015 & 0.6982 \\
\hline 0.2948 & 0.3550 & 0.104 & 0.000 & 0.417 & 0.583 & 0.0999 & 0.0000 & 0.4191 & 0.5809 & 0.1024 & 0.0000 & 0.4173 & 0.5810 \\
\hline 0.4088 & 0.2889 & 0.140 & 0.000 & 0.554 & 0.446 & 0.1422 & 0.0000 & 0.5535 & 0.4465 & 0.1467 & 0.0000 & 0.5480 & 0.4447 \\
\hline 0.5034 & 0.2480 & 0.193 & 0.000 & 0.642 & 0.358 & 0.1923 & 0.0000 & 0.6419 & 0.3581 & 0.1876 & 0.0000 & 0.6342 & 0.3487 \\
\hline
\end{tabular}


Table 6. Continuation

\begin{tabular}{|c|c|c|c|c|c|c|c|c|c|c|c|c|c|}
\hline \multicolumn{14}{|c|}{$n$-hexane (1) - thiophene (2) - B-PG 1:5 (3) } \\
\hline \multicolumn{6}{|c|}{ experiment } & \multicolumn{4}{|c|}{ NRTL } & \multicolumn{4}{|c|}{ UNIQUAC } \\
\hline \multicolumn{2}{|c|}{ feed } & \multicolumn{2}{|c|}{ raffinate } & \multicolumn{2}{|c|}{ extract } & \multicolumn{2}{|c|}{ raffinate } & \multicolumn{2}{|c|}{ extract } & \multicolumn{2}{|c|}{ raffinate } & \multicolumn{2}{|c|}{ extract } \\
\hline$w_{2}$ & $w_{3}$ & $w_{2}$ & $w_{3}$ & $w_{2}$ & $w_{3}$ & $w_{2}$ & $w_{3}$ & $w_{2}$ & $w_{3}$ & $w_{2}$ & $w_{3}$ & $w_{2}$ & $w_{3}$ \\
\hline 0.1148 & 0.5463 & 0.149 & 0.000 & 0.093 & 0.899 & 0.1487 & 0.0006 & 0.0932 & 0.9068 & 0.1503 & 0.0000 & 0.0928 & 0.9071 \\
\hline 0.2097 & 0.5081 & 0.326 & 0.000 & 0.128 & 0.865 & 0.3068 & 0.0002 & 0.1342 & 0.8658 & 0.3089 & 0.0000 & 0.1335 & 0.8663 \\
\hline 0.3124 & 0.4998 & 0.502 & 0.000 & 0.219 & 0.746 & 0.5200 & 0.0001 & 0.2157 & 0.7843 & 0.5211 & 0.0000 & 0.2157 & 0.7834 \\
\hline 0.4067 & 0.5142 & 0.705 & 0.000 & 0.300 & 0.698 & 0.6938 & 0.0001 & 0.3073 & 0.6927 & 0.6925 & 0.0000 & 0.3115 & 0.6861 \\
\hline \multicolumn{14}{|c|}{ n-heptane (1) - thiophene (2) - B-PG 1:5 (3) } \\
\hline \multicolumn{6}{|c|}{ experiment } & \multicolumn{4}{|c|}{ NRTL } & \multicolumn{4}{|c|}{ UNIQUAC } \\
\hline \multicolumn{2}{|c|}{ feed } & \multicolumn{2}{|c|}{ raffinate } & \multicolumn{2}{|c|}{ extract } & \multicolumn{2}{|c|}{ raffinate } & \multicolumn{2}{|c|}{ extract } & \multicolumn{2}{|c|}{ raffinate } & \multicolumn{2}{|c|}{ extract } \\
\hline$w_{2}$ & $w_{3}$ & $w_{2}$ & $w_{3}$ & $w_{2}$ & $w_{3}$ & $w_{2}$ & $w_{3}$ & $w_{2}$ & $w_{3}$ & $w_{2}$ & $w_{3}$ & $w_{2}$ & $w_{3}$ \\
\hline 0.1067 & 0.4283 & 0.162 & 0.000 & 0.038 & 0.962 & 0.1521 & 0.0007 & 0.0424 & 0.9452 & 0.1345 & 0.0046 & 0.0451 & 0.9549 \\
\hline 0.2006 & 0.3850 & 0.268 & 0.000 & 0.113 & 0.887 & 0.2859 & 0.0005 & 0.1077 & 0.8880 & 0.2878 & 0.0036 & 0.1056 & 0.8944 \\
\hline 0.3041 & 0.3390 & 0.406 & 0.000 & 0.151 & 0.849 & 0.4091 & 0.0003 & 0.1507 & 0.8463 & 0.3982 & 0.0029 & 0.1541 & 0.8459 \\
\hline 0.4096 & 0.2811 & 0.527 & 0.000 & 0.188 & 0.812 & 0.5205 & 0.0002 & 0.1934 & 0.8043 & 0.5251 & 0.0022 & 0.1887 & 0.8113 \\
\hline \multicolumn{14}{|c|}{$i$-octane (1) - thiophene (2) - B-PG 1:5 (3) } \\
\hline \multicolumn{6}{|c|}{ experiment } & \multicolumn{4}{|c|}{ NRTL } & \multicolumn{4}{|c|}{ UNIQUAC } \\
\hline & & raffi & ate & ext & act & raff & ate & ext & act & raff & late & & act \\
\hline$w_{2}$ & $w_{3}$ & $w_{2}$ & $w_{3}$ & $w_{2}$ & $w_{3}$ & $w_{2}$ & $w_{3}$ & $w_{2}$ & $w_{3}$ & $w_{2}$ & $w_{3}$ & $w_{2}$ & $w_{3}$ \\
\hline 0.1047 & 0.5108 & 0.151 & 0.000 & 0.064 & 0.967 & 0.1512 & 0.0000 & 0.0617 & 0.9383 & 0.1515 & 0.0000 & 0.0616 & 0.9385 \\
\hline 0.2115 & 0.5036 & 0.323 & 0.000 & 0.125 & 0.891 & 0.3194 & 0.0000 & 0.1244 & 0.8756 & 0.3210 & 0.0000 & 0.1237 & 0.8763 \\
\hline 0.3208 & 0.4936 & 0.519 & 0.000 & 0.205 & 0.783 & 0.5159 & 0.0000 & 0.2053 & 0.7948 & 0.5149 & 0.0000 & 0.2060 & 0.7940 \\
\hline 0.4135 & 0.5012 & 0.697 & 0.000 & 0.297 & 0.707 & 0.6910 & 0.0000 & 0.2971 & 0.7029 & 0.6921 & 0.0000 & 0.2963 & 0.7034 \\
\hline & & & & & oluene & thio & ene (2) & 3-PG 1:5 & & & & & \\
\hline & & experi & ent & & & & NF & & & & UNI & UAC & \\
\hline & & raffi & ate & ext & & raff & ate & ext & act & raft & late & & act \\
\hline$w_{2}$ & $w_{3}$ & $w_{2}$ & $w_{3}$ & $w_{2}$ & $w_{3}$ & $w_{2}$ & $w_{3}$ & $w_{2}$ & $w_{3}$ & $w_{2}$ & $w_{3}$ & $w_{2}$ & $w_{3}$ \\
\hline 0.1018 & 0.4420 & 0.181 & 0.000 & 0.002 & 0.998 & 0.1888 & 0.0010 & 0.0000 & 1.0000 & 0.1888 & 0.0010 & 0.0000 & 1.0000 \\
\hline 0.1919 & 0.4099 & 0.311 & 0.000 & 0.029 & 0.971 & 0.3111 & 0.0013 & 0.0287 & 0.9713 & 0.3111 & 0.0013 & 0.0287 & 0.9713 \\
\hline 0.3123 & 0.3092 & 0.435 & 0.000 & 0.062 & 0.938 & 0.4285 & 0.0013 & 0.0647 & 0.9353 & 0.4285 & 0.0013 & 0.0647 & 0.9353 \\
\hline 0.4253 & 0.2475 & 0.549 & 0.000 & 0.098 & 0.902 & 0.5546 & 0.0012 & 0.0946 & 0.9054 & 0.5546 & 0.0012 & 0.0946 & 0.9054 \\
\hline
\end{tabular}

Note: Standard uncertainties u are $u(T)=0.2 \mathrm{~K}, u(p)=5 \mathrm{kPa}, u(w) \leq 0.0006$ for the raffinate phase. Extract phase compositions are not experimental but are calculated from the mass balance equation, producing an estimate of $u(w) \leq 0.005$.

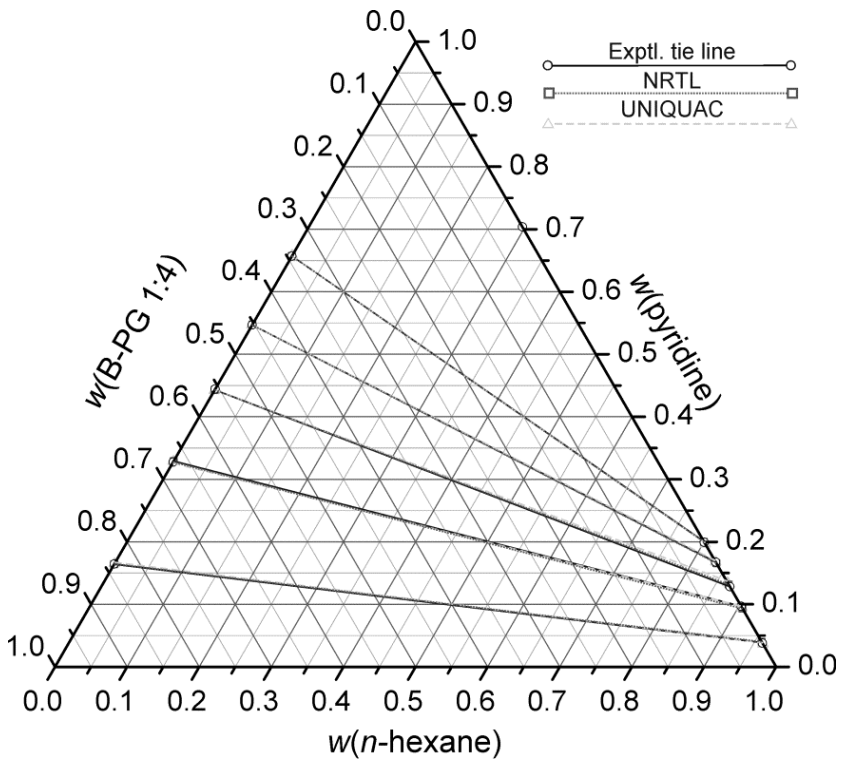

Figure 1. Equilibrium phase compositions in the quasiternary system $n$-hexane (1) - pyridine (2) - B-PG 1:4 (3), at $298.15 \mathrm{~K}$ and at atmospheric pressure.

where superscripts $\mathrm{E}$ and $\mathrm{R}$ denote extract and raffinate phase, respectively. The experiments showed that there are practically no hydrocarbons in the extract

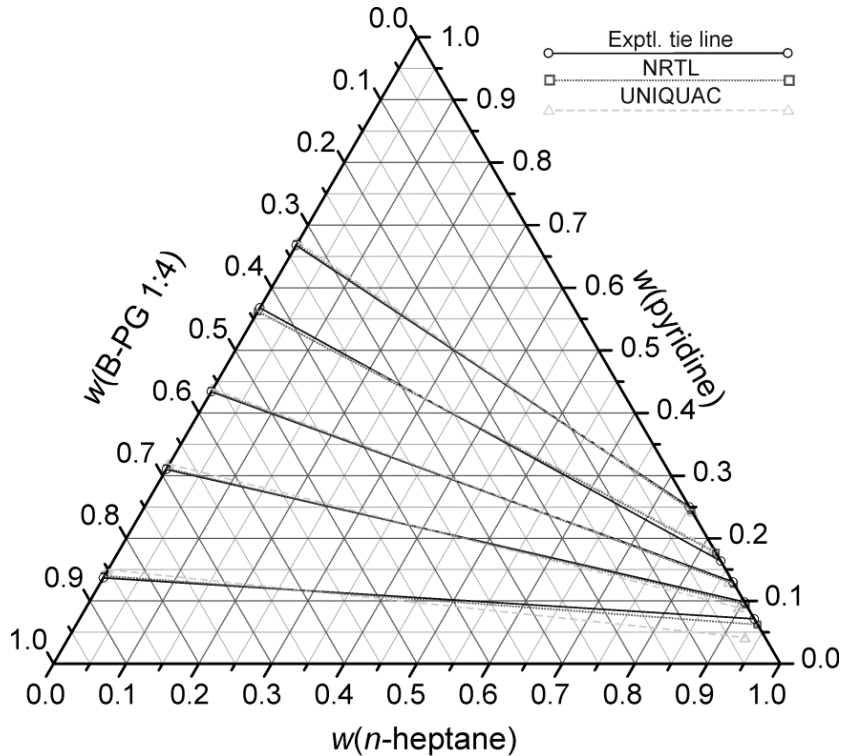

Figure 2. Equilibrium phase compositions in the quasiternary system $n$-heptane (1) - pyridine (2) - B-PG 1:4 (3), at $298.15 \mathrm{~K}$ and at atmospheric pressure.

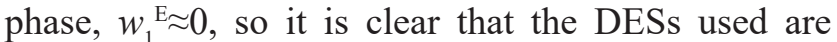
highly selective for pyridine or thiophene, at least in the investigated range of compositions. 


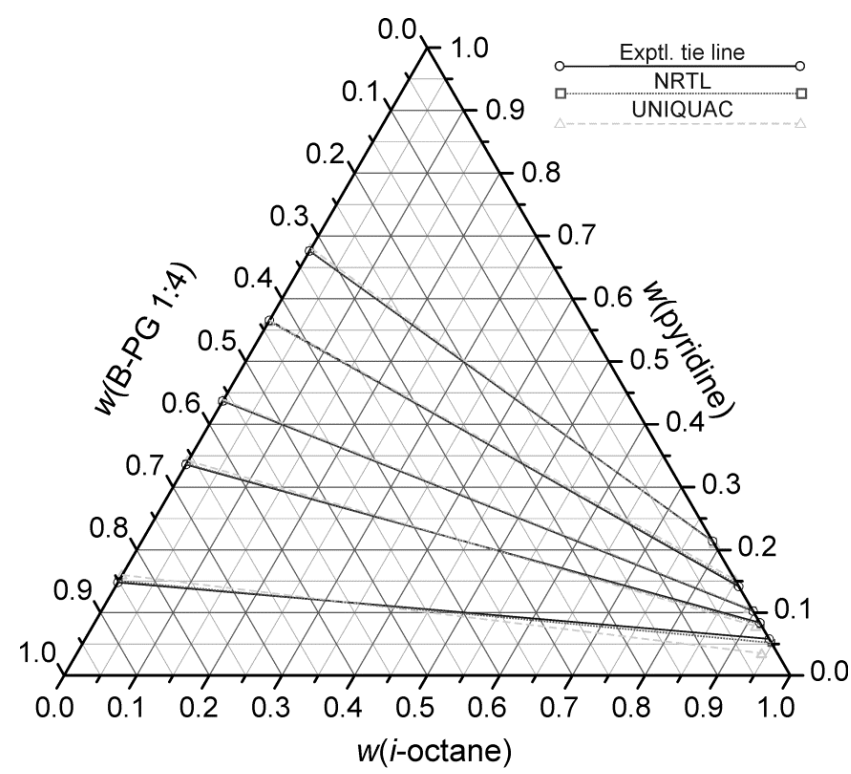

Figure 3. Equilibrium phase compositions in the quasi-ternary system $i$-octane (1) - pyridine (2) - B-PG $1: 4(3)$, at $298.15 \mathrm{~K}$ and at atmospheric pressure.

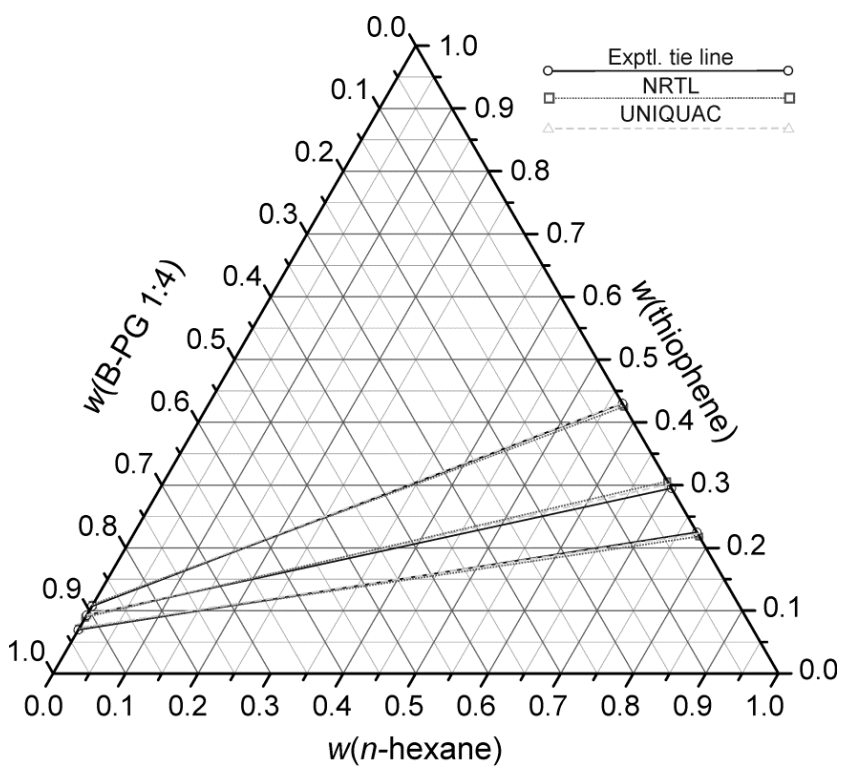

Figure 4. Equilibrium phase compositions in the quasi-ternary system $n$-hexane (1) - thiophene (2) B-PG 1:4 (3), at 298.15 K and at atmospheric pressure.

Distribution ratio is defined by:

$\beta=\frac{w_{2}^{\mathrm{E}}}{w_{2}^{\mathrm{R}}}$

Dependence of the distribution ratio on the mass fraction of pyridine or thiophene in the system as a whole, $w_{2}{ }^{\mathrm{F}}$, is shown in Figure 14. The figure points to relatively high distribution ratios for pyridine systems (1.8 - 4.4) and relatively low distribution ratios for thiophene systems $(<0.6)$. The investigated DESs are therefore more suitable for the removal of nitrogen

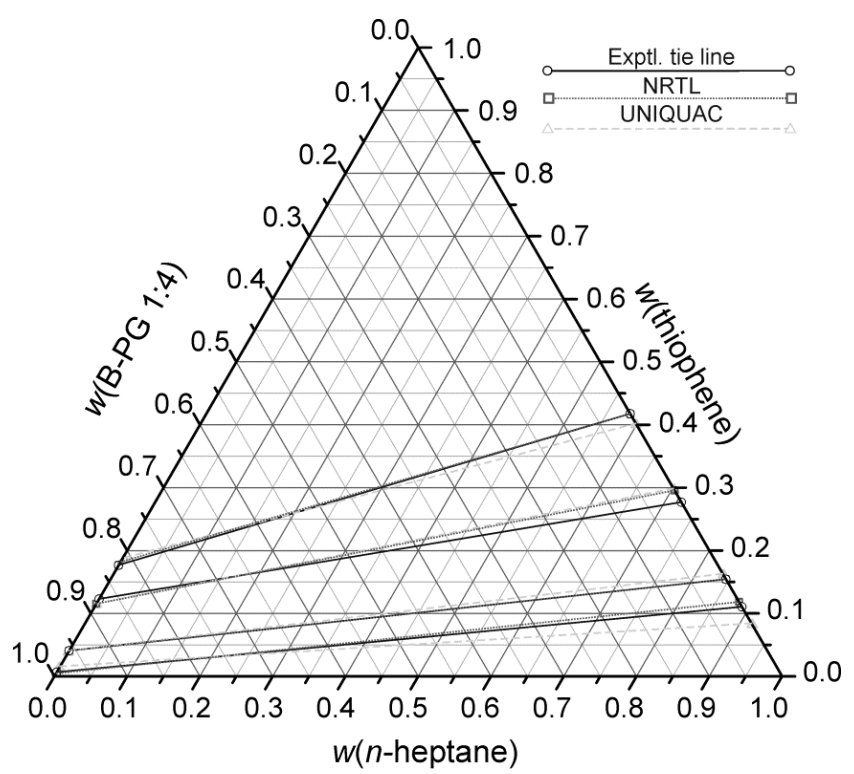

Figure 5. Equilibrium phase compositions in the quasi-ternary system $n$-heptane (1) - thiophene (2) B-PG 1:4 (3), at 298.15 K and at atmospheric pressure.

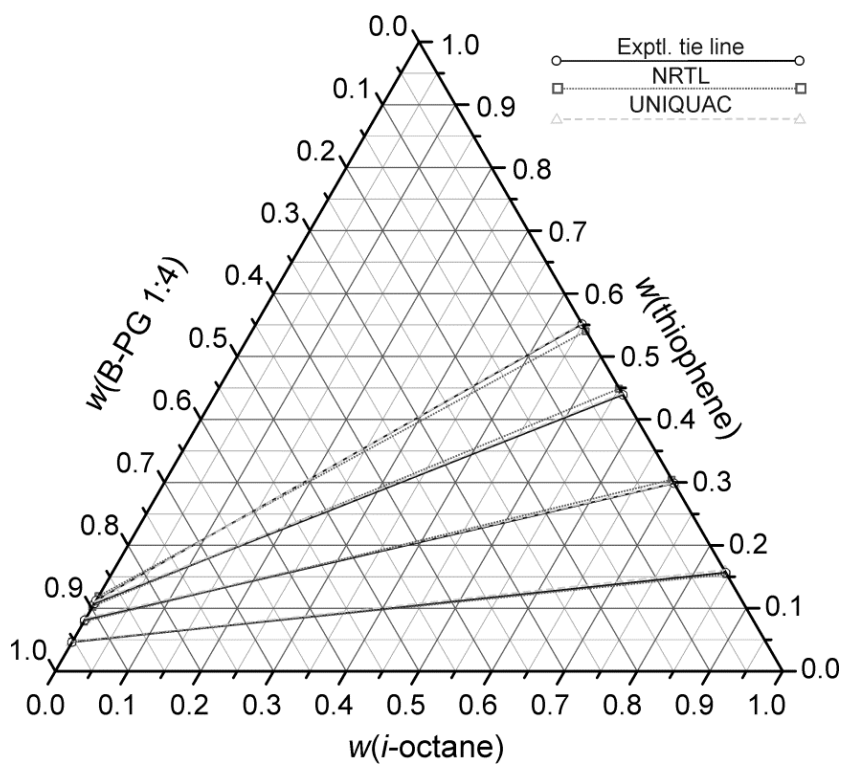

Figure 6. Equilibrium phase compositions in the quasiternary system $i$-octane (1) - thiophene (2) - B-PG 1:4 (3), at 298.15 K and at atmospheric pressure.

compounds (denitrification) in comparison to sulfur compounds (desulfurization). This is similar to the results found for some other DESs, for example those based on glycerol or ethylene glycol in combination with choline chloride (Rogošić and Zagajski Kučan, 2018).

\section{Modeling}

NRTL and UNIQUAC models are well-known models and they are frequently used to describe liquid-liquid phase equilibria in various systems. The NRTL model takes into account local concentrations caused by differences between homogeneous and 


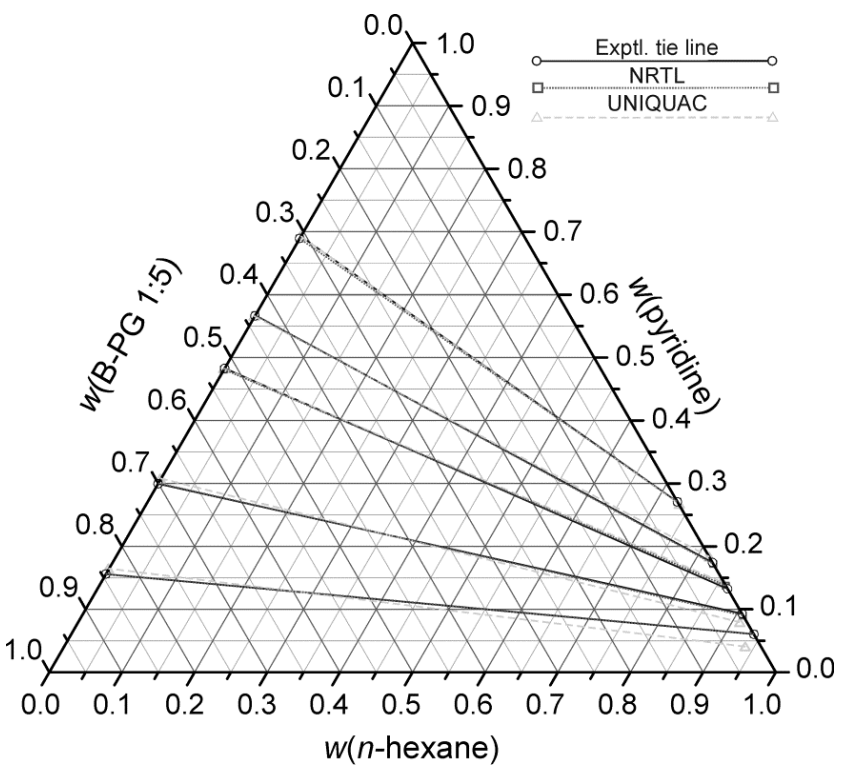

Figure 7. Equilibrium phase compositions in the quasiternary system $n$-hexane (1) - pyridine (2) - B-PG 1:5 (3), at 298.15 K and at atmospheric pressure.

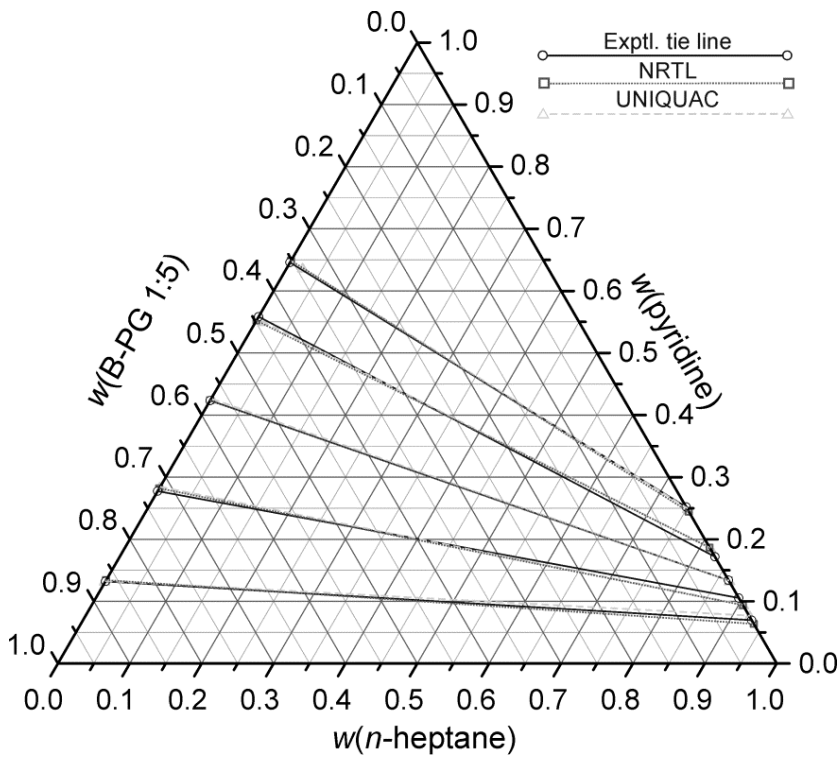

Figure 8. Equilibrium phase compositions in the quasiternary system $n$-heptane (1) - pyridine (2) - B-PG 1:5 (3), at 298.15 K and at atmospheric pressure.

heterogeneous Gibbs interaction energies. Interaction energy parameters are denoted $\tau_{i j}$ and $\tau_{j i}$ for pairs of molecules or other species. The third parameter $\alpha_{i j}=\alpha_{j i}$ is introduced in the model to account for noninteraction (non-random) effects. The excess Gibbs function, $g^{\mathrm{ex}}$, is then calculated by:

$$
\frac{g^{\text {ex }}}{R T}=\sum_{i=1}^{n_{c}} x_{i}\left[\frac{\sum_{j=1}^{n_{c}} \tau_{j i} G_{j i} x_{j}}{\sum_{k=1}^{n_{c}} G_{k i} x_{k}}\right]
$$

with:

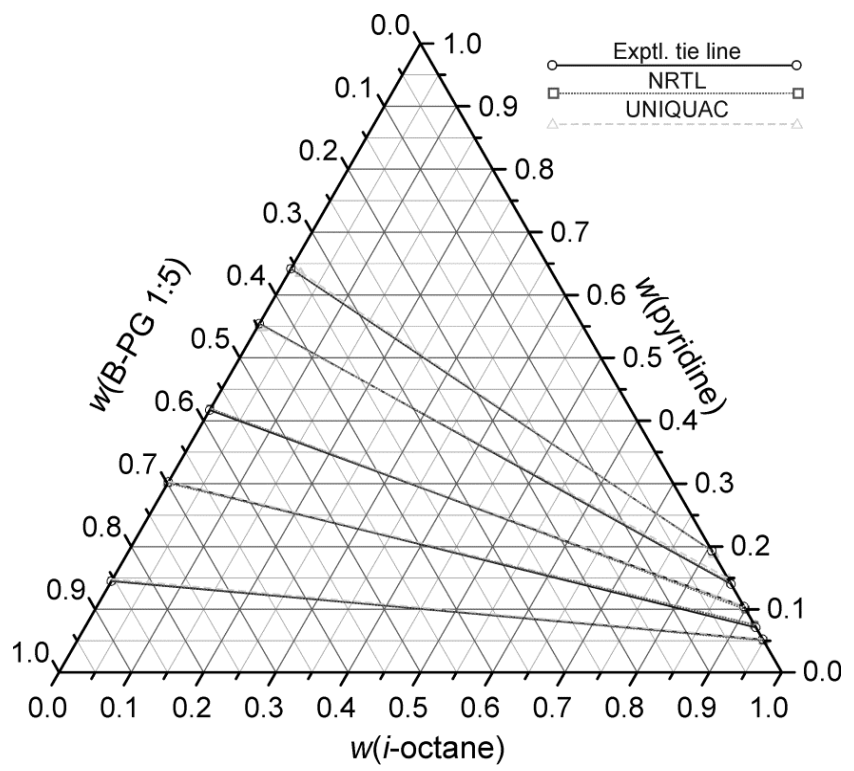

Figure 9. Equilibrium phase compositions in the quasi-ternary system $i$-octane (1) - pyridine (2) - B-PG $1: 5$ (3), at $298.15 \mathrm{~K}$ and at atmospheric pressure.

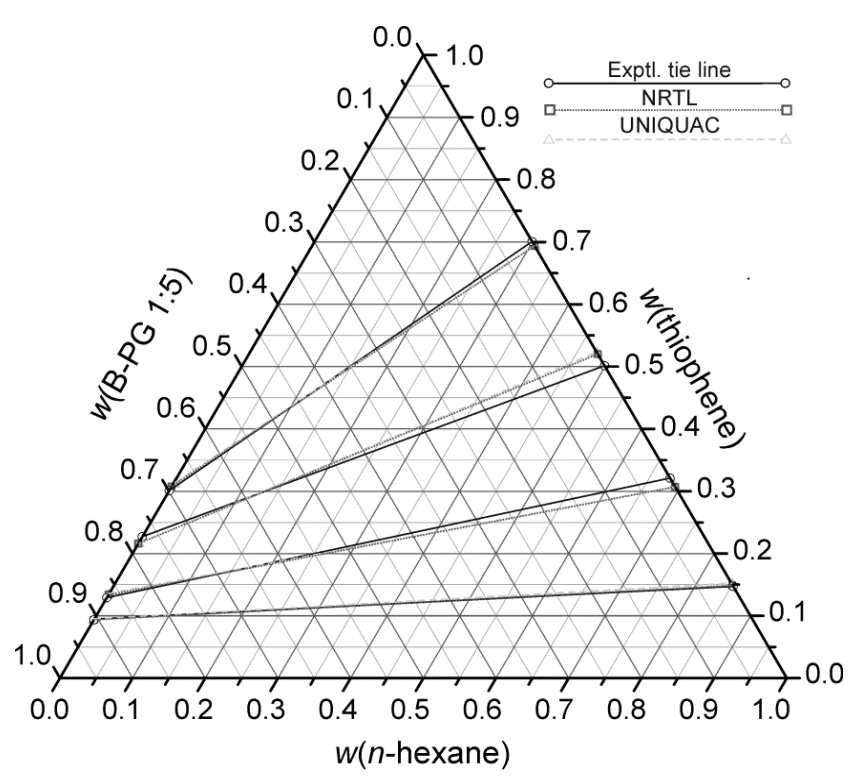

Figure 10. Equilibrium phase compositions in the quasi-ternary system $n$-hexane (1) - thiophene (2) - B-PG 1:5 (3), at 298.15 K and at atmospheric pressure.

$\mathrm{G}_{\mathrm{ij}}=\exp \left(-\alpha_{\mathrm{ij}} \tau_{\mathrm{ij}}\right)$

$n_{\mathrm{c}}$ stands for the number of components. $\alpha$ parameters are commonly fixed, in this work to the value of 0.3 for all the systems. $\tau$ parameters are to be regressed from the experimental tie line data.

The expression for activity coefficients of individual components may be derived simply by calculating the corresponding derivatives of the excess Gibbs function, thus obtaining: 


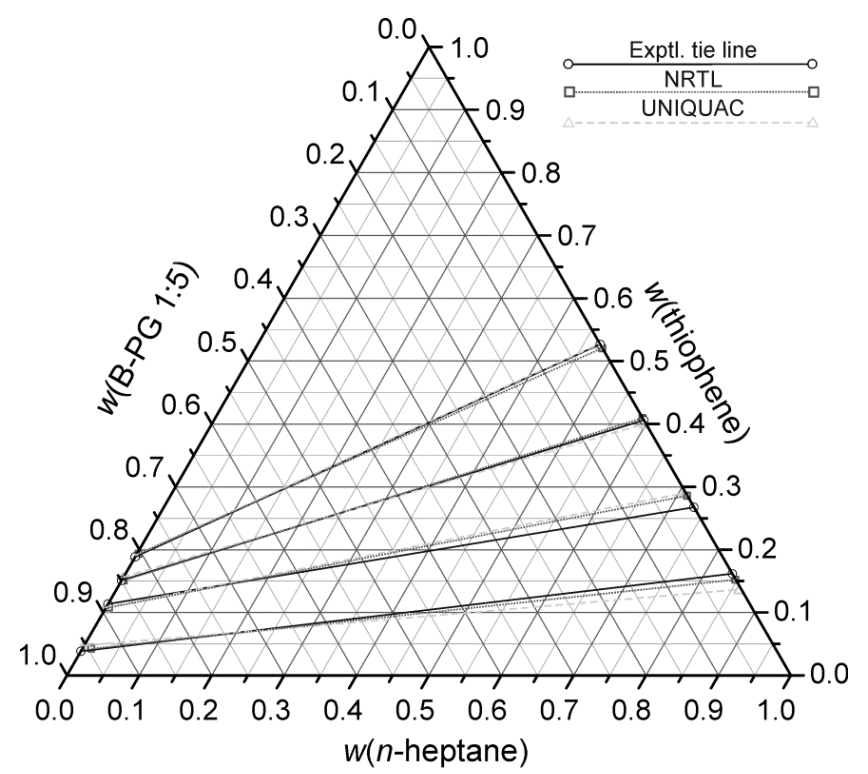

Figure 11. Equilibrium phase compositions in the quasi-ternary system $n$-heptane (1) - thiophene (2) B-PG 1:5 (3), at 298.15 K and at atmospheric pressure.

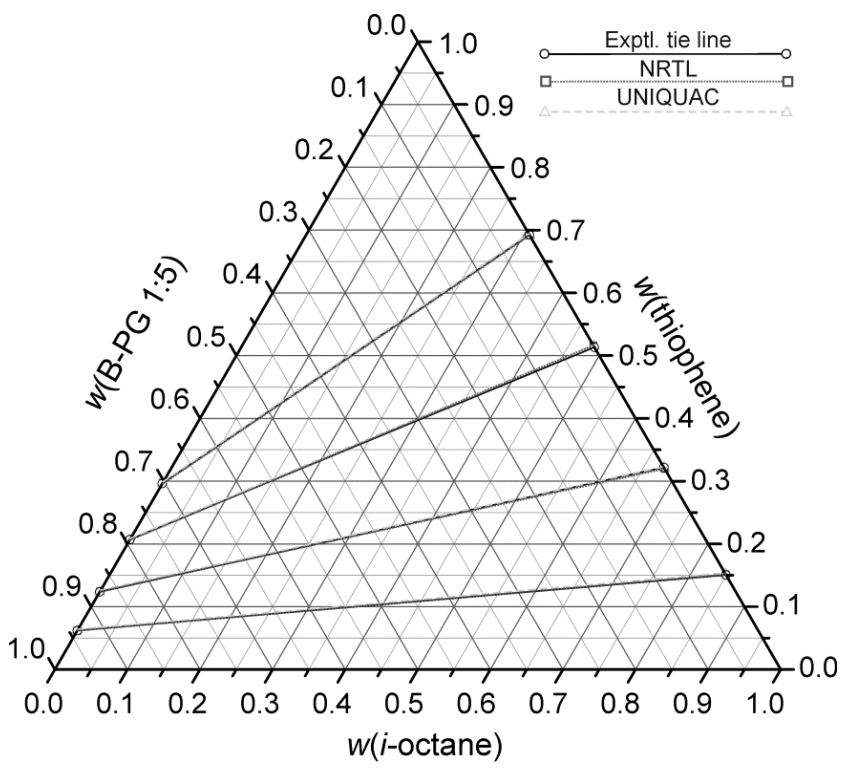

Figure 12. Equilibrium phase compositions in the quasi-ternary system $i$-octane (1) - thiophene (2) - B-PG 1:5 (3), at 298.15 K and at atmospheric pressure.

$$
\ln \gamma_{i}=\frac{\sum_{j=1}^{n_{c}} x_{j} \tau_{j i} G_{j i}}{\sum_{l=1}^{n_{c}} x_{l} G_{l i}}+\sum_{j=1}^{n_{c}} \frac{x_{j} G_{i j}}{\sum_{l=1}^{n_{c}} x_{l} G_{l j}}\left(\tau_{i j}-\frac{\sum_{m=1}^{n_{c}} x_{m} \tau_{m j} G_{m j}}{\sum_{l=1}^{n_{c}} x_{l} G_{l j}}\right)
$$

The UNIQUAC model incorporates two contributions to the excess Gibbs function. The combinatorial one, $g^{\mathrm{ex}, \mathrm{C}}$, accounts for the size and shape differences of molecules or species:

$$
\frac{\mathrm{g}^{\mathrm{ex}, \mathrm{C}}}{\mathrm{RT}}=\sum_{\mathrm{i}=1}^{\mathrm{n}_{\mathrm{c}}} \mathrm{x}_{\mathrm{i}} \ln \frac{\Phi_{\mathrm{i}}}{\mathrm{x}_{\mathrm{i}}}+\frac{\mathrm{z}}{2} \sum_{\mathrm{i}=1}^{\mathrm{n}_{\mathrm{c}}} \mathrm{q}_{\mathrm{i}} \mathrm{x}_{\mathrm{i}} \ln \frac{\Theta_{\mathrm{i}}}{\Phi_{\mathrm{i}}}
$$

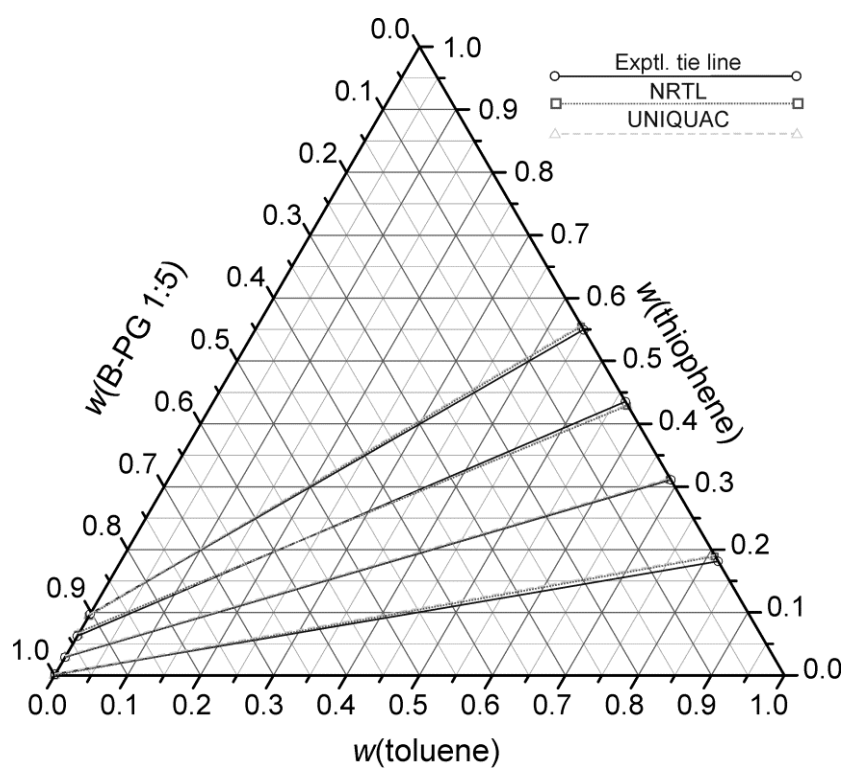

Figure 13. Equilibrium phase compositions in the quasi-ternary system toluene (1) - thiophene (2) B-PG 1:5 (3), at 298.15 K and at atmospheric pressure.

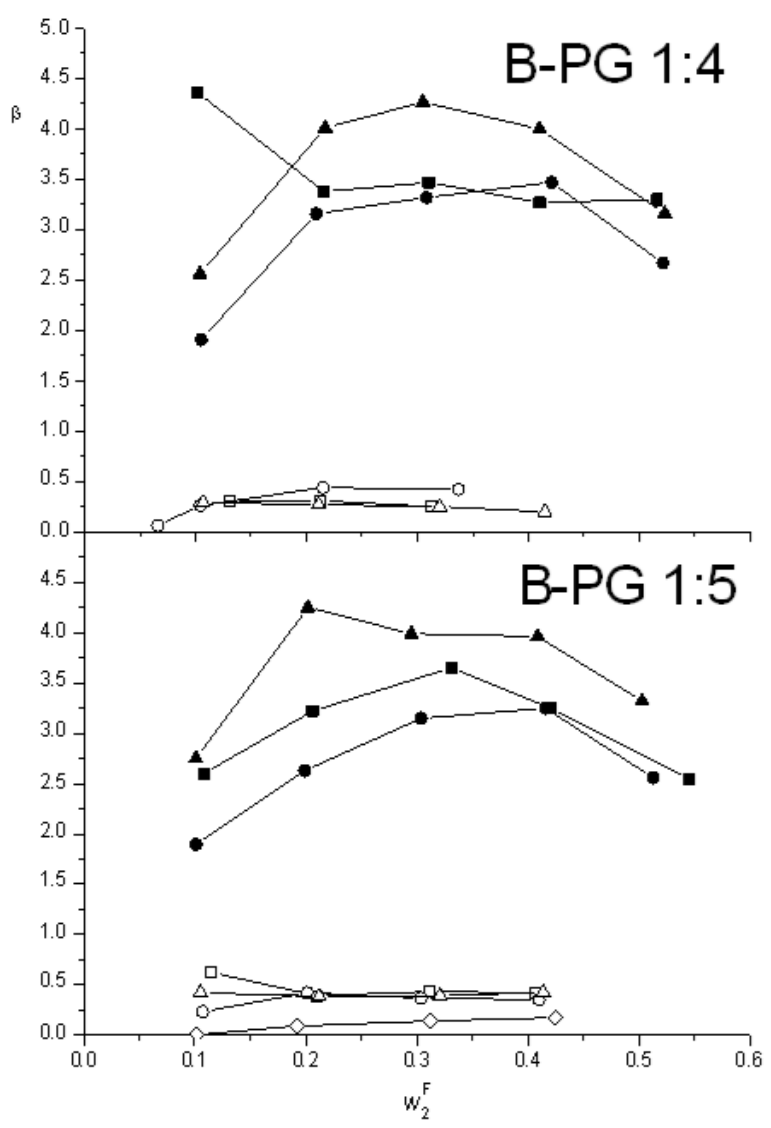

Figure. 14. The dependence of distribution ratio, $\beta$, on the mass fraction of pyridine or thiophene in the feed, $w_{2}{ }^{F}$. Pyridine and thiophene systems are denoted by black and white symbols, respectively. Squares, circles, triangles, and diamonds stand for the systems with $n$-hexane, $n$-heptane, $i$-octane, and toluene, respectively. The measurements were done at 298.15 $\mathrm{K}$ and at atmospheric pressure. 
$z$ is the lattice coordination number. $\Phi_{i}$ and $\Theta_{i}$ are volume and surface fractions of component $i$, respectively. These are calculated from $r_{i}$ and $q_{i}$ volume and surface parameters of the components, respectively, and composition of the mixture expressed in mole fractions $-x_{i}$, using the following formulae:

$$
\begin{gathered}
\Phi_{i}=\frac{\mathrm{x}_{\mathrm{i}} \mathrm{r}_{\mathrm{i}}}{\sum_{\mathrm{j}=1}^{\mathrm{n}_{\mathrm{c}}} \mathrm{x}_{j} \mathrm{r}_{\mathrm{j}}} \\
\Theta_{\mathrm{i}}=\frac{\mathrm{x}_{\mathrm{i}} \mathrm{q}_{\mathrm{i}}}{\sum_{\mathrm{j}=1}^{\mathrm{n}_{\mathrm{c}}} \mathrm{x}_{j} \mathrm{q}_{j}}
\end{gathered}
$$

$r_{i}$ and $q_{i}$ are characteristic parameters for the molecule or species as the whole; however, they are commonly calculated by the group contribution approach using:

$$
\begin{aligned}
& \mathrm{r}_{\mathrm{i}}=\sum_{\mathrm{k}=1}^{\mathrm{n}_{\mathrm{g}}} \mathrm{v}_{\mathrm{ki}} \mathrm{R}_{\mathrm{k}} \\
& \mathrm{q}_{\mathrm{i}}=\sum_{\mathrm{k}=1}^{\mathrm{n}_{\mathrm{g}}} \mathrm{v}_{\mathrm{ki}} \mathrm{Q}_{\mathrm{k}}
\end{aligned}
$$

$R_{k}$ and $Q_{k}$ are the volume and surface parameters of structural group $k$, respectively.

Concerning the structural parameters of the UNIQUAC model, for low-molecular-weight compounds (thiophene, pyridine and hydrocarbons) they were determined using the characteristic structural group parameters tabulated in the literature, e.g., by Magnussen et al. (1981). For high-molecularweight compounds, ions or quasi-components like DESs studied in this article other approaches might be appropriate. For DESs as quasi-components, the approach similar to the one of Domańska (1989) is suggested in this paper. According to this simple correlation, volume $\left(r_{i}\right)$ and surface $\left(q_{i}\right)$ parameters are linked with molar volumes:

$\mathrm{r}_{\mathrm{i}}=0.029281 \mathrm{v}_{\mathrm{i}}$

$\mathrm{q}_{\mathrm{i}}=\frac{(\mathrm{z}-2) \mathrm{r}_{\mathrm{i}}}{\mathrm{z}}+\frac{2\left(1-\mathrm{l}_{\mathrm{i}}\right)}{\mathrm{z}}$

Molar volumes, $v_{i}$, can be calculated easily from the measured densities and molar masses of the quasi-components. The "bulk" factor $l_{i}$ and lattice coordination number, $z$, were set to 0 and 10 , respectively. UNIQUAC structural parameters used here are presented in Table 7.

The residual contribution, $g^{\text {ex,R }}$, describes the interaction between molecules:
Table 7. UNIQUAC structural parameters.

\begin{tabular}{ccc}
\hline Component & $\boldsymbol{r}$ & $\boldsymbol{q}$ \\
\hline$n$-hexane & 4.4998 & 3.856 \\
$n$-heptane & 5.1742 & 4.396 \\
$i$-octane & 5.8463 & 5.008 \\
toluene & 3.9228 & 2.968 \\
thiophene & 2.8569 & 2.140 \\
pyridine & 2.9993 & 2.113 \\
B-PG 1:4 & 11.6173 & 9.494 \\
B-PG 1:5 & 13.6133 & 11.091 \\
\hline
\end{tabular}

$\frac{g^{e x, R}}{R T}=-\sum_{i=1}^{n_{c}} q_{i} x_{i} \ln \left(\sum_{j=1}^{n_{c}} \Theta_{j} \tau_{j i}\right)$

The two adjustable interaction parameters (per pair of components), $\tau_{i j}$ and $\tau_{j i}$, were regressed from tie line data. The overall excess Gibbs energy is calculated simply by summing the two contributions

$\frac{\mathrm{g}^{\text {ex }}}{\mathrm{RT}}=\frac{\mathrm{g}^{\mathrm{ex}, \mathrm{C}}}{\mathrm{RT}}+\frac{\mathrm{g}^{\mathrm{ex}, \mathrm{R}}}{\mathrm{RT}}$

The corresponding expressions for activity coefficients read:

$\ln \gamma_{i}^{C}=\ln \frac{\Phi_{i}}{x_{i}}+\frac{z}{2} q_{i} \ln \frac{\Theta_{i}}{\Phi_{i}}+l_{i}-\frac{\Phi_{i}}{x_{i}} \sum_{j=1}^{n_{c}} x_{j} 1_{j}$

$\ln \gamma_{i}^{R}=q_{i}\left(1-\ln \sum_{j=1}^{n_{c}} \Theta_{j} \tau_{j i}-\sum_{j=1}^{n_{c}} \frac{\Theta_{j} \tau_{i j}}{\sum_{k=1}^{n_{c}} \Theta_{k} \tau_{k j}}\right)$

$\ln \gamma_{i}=\ln \gamma_{i}^{C}+\ln \gamma_{i}^{R}$

The modified procedure of Sørensen and Arlt (1979) was applied for finding NRTL and UNIQUAC interaction parameters. The first step was to look for a minimum of the function:

$\mathrm{OF}_{1}=\sum_{\mathrm{j}=1}^{\mathrm{n}_{\mathrm{d}}} \sum_{\mathrm{i}=1}^{\mathrm{n}_{\mathrm{c}}}\left(\frac{\mathrm{x}_{\mathrm{i}}^{\mathrm{R}} \gamma_{\mathrm{i}}^{\mathrm{R}}-\mathrm{x}_{\mathrm{i}}^{\mathrm{E}} \gamma_{\mathrm{i}}^{\mathrm{E}}}{\mathrm{x}_{\mathrm{i}}^{\mathrm{R}} \gamma_{\mathrm{i}}^{\mathrm{R}}+\mathrm{x}_{\mathrm{i}}^{\mathrm{E}} \gamma_{\mathrm{i}}^{\mathrm{E}}}\right)_{j}^{2}+\mathrm{Q}\left(\tau_{12}^{2}+\tau_{21}^{2}+\tau_{13}^{2}+\tau_{31}^{2}+\tau_{23}^{2}+\tau_{32}^{2}\right)$

$n_{\mathrm{d}}$ and $n_{\mathrm{c}}=3$ are the numbers of quasi-components and tie lines, respectively. Six interaction parameters, $\tau_{i j}$, were fitted. The nonrandomness parameter values of the NRTL model were all set to $\alpha_{i j}=0.3$. The expressions for the coefficients of activity, $\gamma_{i}$ were derived by differentiating the corresponding equations for the excess Gibbs energy. For both models $Q$-values (penalty function values) were set to $1 \times 10^{-6}$ according to Casal (2010).

In the second step the function: 


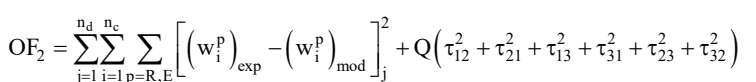

was minimised with respect to $\tau_{i j}$, using values calculated in the first step for the initiation of the fitting procedure. The $\tau_{i j}$ values are not explicit in [Eq. (4)] but involved in the procedure used for the calculation of $\left(w_{i}^{p}\right)_{\bmod }$. The function $O F_{2}$ is aimed at obtaining the best possible agreement of experimental (exp) and model (mod) values of equilibrium compositions with mass fractions, $w_{i}$, as the composition variables. (The original Sørensen-Arlt procedure uses mole fractions in the definition of $O F_{2}$. Nevertheless, mole fractions are always used - as they should be - in the expressions for $\gamma_{i}$ that are included in the calculation subroutines.) $p$ stands for the raffinate (R) or extract (E). Here, $Q$-values of $1 \times 10^{-10}$ were used both for NRTL and UNIQUAC, again according to Casal (2010).

Optimal interaction parameters of the models are shown in Tables 8 and 9. The tables also contain the average absolute prediction errors expressed over equilibrium mass fractions of components, calculated according to:

$$
\mathrm{A}=\sqrt{\frac{\mathrm{OF}_{2}-\mathrm{Q}\left(\tau_{12}^{2}+\tau_{21}^{2}+\tau_{13}^{2}+\tau_{31}^{2}+\tau_{23}^{2}+\tau_{32}^{2}\right)}{\mathrm{n}_{\mathrm{d}} \cdot \mathrm{n}_{\mathrm{c}} \cdot 2}}
$$

Somewhat smaller $A$-values were obtained for the NRTL model $(\bar{A}=0.0043$ and 0.0051 for NRTL and UNIQUAC, respectively). The model tie lines are included in Table 6 and in Figures 1 - 13. The models seem to describe experimental data well. The maximum error for UNIQUAC is $A=0.0101$ for the $n$-heptane (1) - thiophene (2) - B-PG 1:5 (3) system. For NRTL, the maximum error is found with the $n$-hexane (1) - thiophene (2) - B-PG 1:5 (3) system and amounts to $A=0.0082$.

Binary interaction parameters determined in quasiternary systems could be used in principle for the description of multicomponent systems as well, for example those described in Rogošić and Zagajski Kučan (2018). However, it should be noted that despite the good description of the experimental data in quasithree-component systems, the parameter values are very loosely related to the true interactions in the system. Specifically, the mass fractions of hydrocarbons

Table 8. Optimal parameters of the NRTL and UNIQUAC activity coefficient models and the average absolute prediction errors for the systems with DES B-PG 1:4.

\begin{tabular}{lccccccc}
\hline \multicolumn{1}{c}{ NRTL $\boldsymbol{\alpha}_{\mathbf{1 2}} ; \boldsymbol{\alpha}_{\mathbf{1 3}} ; \boldsymbol{\alpha}_{\mathbf{2 3}}=\mathbf{0 . 3} \mathbf{0 . 3} ; \mathbf{0 . 3}$} & $\boldsymbol{\tau}_{\mathbf{1 2}}$ & $\boldsymbol{\tau}_{\mathbf{1 3}}$ & $\boldsymbol{\tau}_{\mathbf{2 1}}$ & $\boldsymbol{\tau}_{\mathbf{2 3}}$ & $\boldsymbol{\tau}_{\mathbf{3 1}}$ & $\boldsymbol{\boldsymbol { \tau } _ { \mathbf { 3 2 } }}$ & $\boldsymbol{A}$ \\
\hline$n$-hexane (1) - pyridine (2) - B-PG 1:4 (3) & 1.5952 & 19.7077 & 7.9844 & 21.5525 & 6.4896 & -1.1851 & 0.0016 \\
$n$-heptane (1) - pyridine (2) - B-PG 1:4 (3) & 2.1772 & 11.4895 & 9.7467 & 21.6871 & 18.2586 & 2.5599 & 0.0050 \\
$i$-octane (1) - pyridine (2) - B-PG 1:4 (3) & 2.2532 & 12.0000 & 9.4107 & 18.4315 & 18.5736 & 2.4763 & 0.0021 \\
$n$-hexane (1) - thiophene (2) - B-PG 1:4 (3) & 1.5936 & 12.7732 & 13.7979 & 8.3304 & 20.5367 & 10.6499 & 0.0048 \\
$n$-heptane (1) - thiophene (2) - B-PG 1:4 (3) & 5.2635 & 15.0755 & 12.0013 & 13.8160 & 12.1866 & 4.2244 & 0.0063 \\
$i$-octane (1) - thiophene (2) - B-PG 1:4 (3) & 1.5487 & 13.4831 & 14.6053 & 7.5884 & 21.1505 & 10.3957 & 0.0053 \\
\hline \multicolumn{1}{c}{ UNIQUAC } & $\boldsymbol{\tau}_{\mathbf{1 2}}$ & $\boldsymbol{\tau}_{\mathbf{1 3}}$ & $\boldsymbol{\tau}_{\mathbf{2 1}}$ & $\boldsymbol{\tau}_{\mathbf{2 3}}$ & $\boldsymbol{\tau}_{\mathbf{3 1}}$ & $\boldsymbol{\boldsymbol { \tau } _ { \mathbf { 3 2 } }}$ & $\boldsymbol{A}$ \\
\hline n-hexane (1) - pyridine (2) - B-PG 1:4 (3) & 1.3552 & 0.6080 & 0.0245 & 2.7793 & 0.0431 & 0.3972 & 0.0021 \\
$n$-heptane (1) - pyridine (2) - B-PG 1:4 (3) & 1.8391 & 1.5234 & 0.0505 & 0.2670 & 0.0597 & 4.2797 & 0.0088 \\
$i$-octane (1) - pyridine (2) - B-PG 1:4 (3) & 1.7755 & 1.5587 & 0.0725 & 0.2844 & 0.0515 & 4.4658 & 0.0062 \\
$n$-hexane (1) - thiophene (2) - B-PG 1:4(3) & 0.8637 & 0.4563 & 0.0765 & 0.1729 & 0.0589 & 0.7295 & 0.0022 \\
n-heptane (1) - thiophene (2) - B-PG 1:4(3) & 3.9416 & 0.1249 & 0.0018 & 3.0683 & 0.0756 & 0.1042 & 0.0033 \\
$i$-octane (1) - thiophene (2) - B-PG 1:4 (3) & 1.1483 & 0.4315 & 0.0854 & 0.1168 & 0.1219 & 0.8969 & 0.0007 \\
\hline
\end{tabular}

Table 9. Optimal parameters of the NRTL and UNIQUAC activity coefficient models and the average absolute prediction errors for the systems with DES B-PG 1:5.

\begin{tabular}{lccccccc}
\hline \multicolumn{1}{c}{ NRTL $\boldsymbol{\alpha}_{\mathbf{1 2}} ; \boldsymbol{\alpha}_{\mathbf{1 3}} ; \boldsymbol{\alpha}_{\mathbf{2 3}}=\mathbf{0 . 3} \mathbf{0 . 3} \mathbf{0 . 3}$} & $\boldsymbol{\tau}_{\mathbf{1 2}}$ & $\boldsymbol{\tau}_{\mathbf{1 3}}$ & $\boldsymbol{\tau}_{\mathbf{2 1}}$ & $\boldsymbol{\tau}_{\mathbf{2 3}}$ & $\boldsymbol{\tau}_{\mathbf{3 1}}$ & $\boldsymbol{\boldsymbol { \tau } _ { \mathbf { 3 2 } }}$ \\
\hline$n$-hexane (1) - pyridine (2) - B-PG 1:5 (3) & 2.3561 & 18.4758 & 9.6071 & 19.9005 & 5.1791 & 1.4430 & 0.0017 \\
$n$-heptane (1) - pyridine (2) - B-PG 1:5 (3) & 2.1756 & 11.4651 & 9.6896 & 21.7230 & 18.2642 & 2.5674 & 0.0059 \\
$i$-octane (1) - pyridine (2) - B-PG 1:5 (3) & 2.3042 & 12.0173 & 9.3955 & 18.4660 & 18.5811 & 2.4770 & 0.0019 \\
$n$-hexane (1) - thiophene (2) - B-PG 1:5 (3) & 10.3862 & 12.4890 & 14.1446 & 10.9529 & 9.4259 & -1.3641 & 0.0082 \\
$n$-heptane (1) - thiophene (2) - B-PG 1:5(3) & 1.1517 & 7.4370 & 12.0344 & 10.5992 & 2.9090 & 9.8906 & 0.0081 \\
$i$-octane (1) - thiophene (2) - B-PG 1:5 (3) & 0.1830 & 14.3038 & 16.2916 & 12.6268 & 10.6391 & -0.7011 & 0.0014 \\
toluene (1) - thiophene (2) - B-PG 1:5 (3) & 0.5731 & 8.7294 & 13.2245 & 7.7782 & 21.1405 & 9.7197 & 0.0037 \\
\hline \multicolumn{1}{c}{ UNIQUAC } & $\boldsymbol{\tau}_{\mathbf{1 2}}$ & $\boldsymbol{\tau}_{\mathbf{1 3}}$ & $\boldsymbol{\tau}_{\mathbf{2 1}}$ & $\boldsymbol{\tau}_{\mathbf{2 3}}$ & $\boldsymbol{\boldsymbol { \tau } _ { \mathbf { 3 1 } }}$ & $\boldsymbol{\boldsymbol { \tau } _ { \mathbf { 3 2 } }}$ & $\boldsymbol{A}$ \\
\hline$n$-hexane (1) - pyridine (2) - B-PG 1:5 (3) & 2.1026 & 1.5246 & 0.0243 & 0.1822 & 0.0559 & 5.8425 & 0.0062 \\
$n$-heptane (1) - pyridine (2) - B-PG 1:5 (3) & 0.2400 & 0.0444 & 1.3738 & 2.3506 & 0.0041 & 0.0038 & 0.0074 \\
$i$-octane (1) - pyridine (2) - B-PG 1:5 (3) & 0.2250 & 0.0480 & 1.4369 & 2.5417 & 0.0041 & 0.0037 & 0.0046 \\
$n$-hexane (1) - thiophene (2) - B-PG 1:5(3) & 0.0998 & 0.0025 & 2.5325 & 0.0021 & 0.0503 & 2.8164 & 0.0080 \\
$n$-heptane (1) - thiophene (2) - B-PG 1:5(3) & 1.0880 & 1.3835 & 0.0248 & 0.3163 & 0.0063 & 0.7248 & 0.0101 \\
$i$-octane (1) - thiophene (2) - B-PG 1:5 (3) & 0.2473 & 0.0029 & 2.3157 & 0.0022 & 0.0268 & 2.6582 & 0.0014 \\
toluene (1) - thiophene (2) - B-PG 1:5 (3) & 0.2320 & 0.0033 & 4.7599 & 0.0016 & 0.0871 & 2.2170 & 0.0053 \\
\hline
\end{tabular}


in the extract or the mass fractions of DES in the raffinate were under the detection limit. Hence, their values were set to 0 or - to be more precise - to a low value of $\sim 10^{-6}$ which was chosen arbitrarily to avoid the division-by-zero computation error. Therefore it should be recognised that the resulting interaction parameters contain arbitrarily assigned information. The problem can be resolved only by envisaging some other experimental method. For example, ${ }^{1} \mathrm{H}$ NMR might be used for measuring very low solubility values of hydrocarbons in DES (Hizaddin et al., 2014). An alternative is the gas-liquid chromatography (GLC) method used for studying interactions at very low solubility levels. The method is suitable for nonvolatile solvents and therefore successfully applied in ionic liquids (Kato and Gmehling, 2004; Nebig et al., 2009; Domańska and Laskowska, 2009; Letcher et al., 2009; Olivier et al., 2010) and DESs (Verevkin et al., 2015). The infinite dilution activity coefficients are determined as the carriers of the key experimental information.

\section{CONCLUSIONS}

This paper describes the preparation and characterization of two selected DESs based on betaine and propylene glycol in molar proportions of 1:4 and 1:5. Refractive index, thermal conductivity, temperature diffusivity, heat capacity, specific conductance, density and dynamic viscosity were all determined for the prepared eutectic solvents. Also, the applicability of the prepared DESs for the extractive purification of nitrogen and sulfur-containing substances from hydrocarbons was explored. For this purpose, model systems were investigated. LLE were experimentally determined in 13 quasi-ternary mixtures with hydrocarbons (aliphatic or aromatic, $n$-hexane, $n$-heptane, $i$-octane or toluene) as component 1 , pyridine or thiophene as component 2 and DES (BPG 1:4 or B-PG 1:5) as component 3 at the temperature of $298.15 \mathrm{~K}$ and at atmospheric pressure. The distribution ratios of pyridine or thiophene between the phases were calculated and DESs were recognised as potential agents for denitrification rather than for desulfurization. It was assumed that DESs could be viewed as quasi-components, which allowed NRTL and UNIQUAC activity coefficient model parameters to be optimised for the description of the experimental LLE. A fair agreement of model and experiments was found, particularly with NRTL.

\section{ACKNOWLEDGEMENT}

This work was supported by the Croatian Science Foundation, under the project Green Solvents for Green Technologies (9550). The authors acknowledge the contribution of the laboratories of INA Sisak oil refinery in determining the densities of the investigated DESs.

\section{NOMENCLATURE}

\begin{tabular}{|c|c|}
\hline$A$ & Average absolute prediction error \\
\hline$a$ & Temperature diffusivity $\left[\mathrm{mm}^{2} \mathrm{~s}^{-1}\right]$ \\
\hline B & Betaine \\
\hline & Heat capacity $\left[\mathrm{J}\left(\mathrm{g} \mathrm{K}^{-1}\right]\right.$ \\
\hline COOSMO-RS & $\begin{array}{l}\text { Conductor-like Screening MOdel for } \\
\text { Realistic Solvents }\end{array}$ \\
\hline DES & Deep eutectic solvent \\
\hline$g^{\text {ex }}$ & Excess molar Gibbs energy $\left[\mathrm{J} \mathrm{mol}^{-1}\right]$ \\
\hline$g^{\mathrm{ex}, \mathrm{C}}$ & $\begin{array}{l}\text { Combinatorial part of excess molar } \\
\text { Gibbs energy }\left[\mathrm{J} \mathrm{mol}^{-1}\right]\end{array}$ \\
\hline$G_{i j}$ & $\begin{array}{l}\text { "Composite" parameter of the NRTL } \\
\text { model }\end{array}$ \\
\hline GLC & Gas-liquid chromatography \\
\hline${ }^{1} \mathrm{H}$ NMR & Proton nuclear magnetic resonance \\
\hline HBA & Hydrogen bond acceptor \\
\hline HBD & Hydrogen bond donor \\
\hline HDN & Hydrodenitrification \\
\hline HDS & Hydrodesulfurization \\
\hline IL & Ionic liquid \\
\hline LLE & Liquid-liquid equilibrium \\
\hline$l_{i}$ & "Bulk" factor of the UNIQUAC model \\
\hline$n_{\mathrm{c}}$ & Number of components \\
\hline$n_{\mathrm{g}}$ & Overall number of structural group \\
\hline & Refractive index \\
\hline NRTL & Non-random two-liquid model \\
\hline PG & Propylene glycol \\
\hline$R$ & Gas constant, $8.314 \mathrm{~J} \mathrm{~K}^{-1} \mathrm{~mol}^{-1}$ \\
\hline$Q$ & Penalty function value \\
\hline$q_{i}$ & $\begin{array}{l}\text { Surface parameter of the UNIQUAC } \\
\text { model }\end{array}$ \\
\hline$Q_{k}$ & Surface parameter of structural group \\
\hline$R^{2}$ & Regression coefficient \\
\hline$r_{i}$ & $\begin{array}{l}\text { Volume parameter of the UNIQUAC } \\
\text { model }\end{array}$ \\
\hline$R_{k}$ & Volume parameter of structural group \\
\hline$S^{k}$ & Selectivity \\
\hline$T$ & Temperature $[\mathrm{K}]$ \\
\hline UNIQUAC & UNIversal QUAsiChemical model \\
\hline$v_{i}$ & Molar volume $\left[\mathrm{m}^{3} \mathrm{~mol}^{-1}\right]$ \\
\hline$w_{i}$ & Mass fraction \\
\hline$x_{i}$ & Mole fraction \\
\hline$z$ & Lattice coordination number \\
\hline$\alpha_{i j}$ & $\begin{array}{l}\text { Nonrandomness parameter of the } \\
\text { NRTL model }\end{array}$ \\
\hline$\beta$ & Distribution ratio \\
\hline$\gamma_{I}$ & Activity coefficient \\
\hline$\Theta_{i}$ & Surface fraction \\
\hline$\lambda^{t}$ & Thermal conductivity $\left[\mathrm{W}(\mathrm{m} \mathrm{K})^{-1}\right]$ \\
\hline$\mu$ & Dynamic viscosity [Pa s] \\
\hline$v_{k i}$ & $\begin{array}{l}\text { Number of structural groups } k \text { in } \\
\text { component } i\end{array}$ \\
\hline
\end{tabular}




$\begin{array}{ll}\rho & \text { Density }\left[\mathrm{kg} \mathrm{m}^{-3}\right] \\ \sigma & \text { Specific conductance }\left[\mu \mathrm{S} \mathrm{cm}^{-1}\right] \\ \tau_{i j} & \text { Interaction parameter of the NRTL } \\ \Phi_{i} & \text { and UNIQUAC models }\end{array}$

\section{REFERENCES}

Abbott, A. P., Cullis, P. M., Gibson, M. J., Harris, R. C., Raven, E., Extraction of glycerol from biodiesel into a eutectic based ionic liquid. Green Chem., 9, 868-872 (2007). https://doi.org/10.1039/b702833d

Ali, M. C., Yang, Q., Fine, A. A., Jin, W., Zhang, Z., Xing, H., Ren, Q., Efficient removal of both basic and non-basic nitrogen compounds from fuels by deep eutectic solvents. Green Chem., 18, 157-164 (2016). https://doi.org/10.1039/C5GC01823D

Casal, M. F. Desulfurization of fuel oils by solvent extraction with ionic liquids, Ph.D. Thesis, University of Santiago de Compostela (2010).

Domańska, U., Laskowska, M., Measurements of activity coefficients at infinite dilution of aliphatic and aromatic hydrocarbons, alcohols, thiophene, tetrahydrofuran, MTBE, and water in ionic liquid [BMIM] $\mathrm{SCN}]$ using GLC. J. Chem. Thermodyn., 41, 645-650 (2009). https://doi.org/10.1016/j. jct.2008.12.018

Domańska, U. Solubility of $n$-alkanols $\left(\mathrm{C}_{16}, \mathrm{C}_{18}, \mathrm{C}_{20}\right)$ in binary solvent mixtures. Fluid Phase Equilib., 46, 223-248 (1989). https://doi.org/10.1016/03783812(89) $80037-8$

Gano, Z. S., Mjalli, F. S., Al-Wahaibi, T., Al-Wahaibi, Y., AlNashef, I. M., Extractive desulfurization of liquid fuel with $\mathrm{FeCl}_{3}$-based deep eutectic solvents: Experimental design and optimization by centralcomposite design. Chem. Eng. Process., 93, 10-20 (2015). https://doi.org/10.1016/j.cep.2015.04.001

Hizaddin, H. F., Hadj-Kali, M. K., Ramalingam, A., Hashim, M. A. Extractive denitrogenation of diesel fuel using ammonium- and phosphonium-based deep eutectic solvents. J. Chem. Thermodyn., 95, 164-173 (2016). https://doi.org/10.1016/j. jct.2015.12.009

Hizaddin, H. F., Ramalingam, A., Ali Hashim, M., Hadj-Kali, M. K. O. Evaluating the performance of deep eutectic solvents for use in extractive denitrification of liquid fuels by the conductorlike screening model for real solvents. J. Chem. Eng. Data, 59, 3470-3487 (2014). https://doi. org/10.1021/je5004302

Hizaddin, H. F., Sarwono, M., Hashim, M. A., Alnashef, I. M., Hadj-Kali, M. K. O., Coupling the capabilities of different complexing agents into deep eutectic solvents to enhance the separation of aromatics from aliphatics. J. Chem. Thermodyn., 84, 67-75 (2015). https://doi.org/10.1016/j. jct.2014.12.024
Jibril, B., Mjalli, F., Naser, J., Gano, Z. New tetrapropylammonium bromide-based deep eutectic solvents: Synthesis and characterizations. J. Mol. Liq., 199, 462-469 (2014). https://doi. org/10.1016/j.molliq.2014.08.004

Kareem, M. A., Mjalli, F. S., Ali Hashim, M., HadjKali, M. K. O., Bagh, F. S. G., Alnashef, I. M. Phase equilibria of toluene/heptane with deep eutectic solvents based on ethyltriphenylphosphonium iodide for the potential use in the separation of aromatics from naphtha. J. Chem. Thermodyn., 65, 138-149 (2013). https://doi.org/10.1016/j.jct.2013.05.046

Kareem, M. A., Mjalli, F. S., Ali Hashima, M., Al Nashef, I. M. Liquid-liquid equilibria for the ternary system (phosphonium based deep eutectic solvent-benzene-hexane) at different temperatures: A new solvent introduced. Fluid Phase Equilib., 314, 52-59 (2012). https://doi.org/10.1016/j. fluid.2011.10.024

Kato, R., Gmehling, J. Activity coefficients at infinite dilution of various solutes in the ionic liquids $[\mathrm{MMIM}]^{+}\left[\mathrm{CH}_{3} \mathrm{SO}_{4}\right]^{-}$, $[\mathrm{MMIM}]^{+}\left[\mathrm{CH}_{3} \mathrm{OC}_{2} \mathrm{H}_{4} \mathrm{SO}_{4}\right]^{-},[\mathrm{MMIM}]^{+}\left[\left(\mathrm{CH}_{3}\right)_{2} \mathrm{PO}_{4}\right]^{-}$, $\left[\mathrm{C}_{5} \mathrm{H}_{5} \mathrm{NC}_{2} \mathrm{H}_{5}\right]^{+}\left[\left(\mathrm{CF}_{3} \mathrm{SO}_{2}\right)_{2} \mathrm{~N}\right]^{-}$and $\left[\mathrm{C}_{5} \mathrm{H}_{5} \mathrm{NH}\right]^{+}\left[\mathrm{C}_{2} \mathrm{H}_{5} \mathrm{OC}_{2} \mathrm{H}_{4} \mathrm{OSO}_{3}\right]^{-}$. Fluid Phase Equilib., 226, 37-44(2004). https://doi.org/10.1016/j. fluid.2004.08.039

Letcher, T. M., Ramjugernath, D., Królikowski, M., Laskowska, M., Naidoo, P., Domańska, U. Activity coefficients at infinite dilution measurements for organic solutes in the ionic liquid $\mathrm{N}$-butyl4-methylpyridinium tosylate using GLC at $T=$ $(328.15,333.15,338.15$, and 343.15)K. Fluid Phase Equilib., 276, 31-36 (2009). https://doi. org/10.1016/j.fluid.2008.10.008

Li, C., Li, D., Zou, S., Li, Z., Yin, J., Wang, A., Cui, Y., Yao, Z., Zhao, Q., Extraction desulfurization process of fuels with ammonium based deep eutectic solvents green chemistry. Green Chem., 15, 2793-2799 (2013). https://doi.org/10.1039/ c3gc41067f

Li, J.-J., Xiao, H., Tang, X.-D., Zhou, M. Green carboxylic acid-based deep eutectic solvents as solvents for extractive desulfurization. Energ. Fuel, 30, 5411-5418 (2016). https://doi.org/10.1021/acs. energyfuels.6b00471

Magnussen, T., Rasmussen, P., Fredenslund, A. UNIFAC parameter table for prediction of liquidliquid equilibria. Ind. Eng. Chem. Process Des. Dev., 20, 331-339 (1981). https://doi.org/10.1021/ i200013a024

Morrison, H. G., Sun, C. C., Neervannan, S. Characterization of thermal behavior ofdeepeutectic solvents and their potential as drug solubilization vehicles. Int. J. Pharm., 378, 136-139 (2009). https://doi.org/10.1016/j.ijpharm.2009.05.039 
Mulyono, S., Hizaddin, H. F., Alnashef, I. M., Hashim, M. A., Fakeeha, A. H., Hadj-Kali, M. $\mathrm{K}$. Separation of BTEX aromatics from $n$-octane using a (tetrabutylammonium bromide + sulfolane) deep eutectic solvent - experiments and COSMORS prediction. RSC Adv., 4, 17597-17606 (2014). https://doi.org/10.1039/c4ra01081g

Nebig, S., Liebert, V., Gmehling, J. Measurement and prediction of activity coefficients at infinite dilution, vapor-liquid equilibria (VLE) and excess enthalpies (HE) of binary systems with 1,1-dialkylpyrrolidinium bis(trifluoromethylsulfonyl)imide using mod. UNIFAC. Fluid Phase Equilib., 277, 6167 (2009). https://doi.org/10.1016/j.fluid.2008.11.013

Olivier, E., Letcher, T. M., Naidoo, P., Ramjugernath, D. Activity coefficients at infinite dilution of organic solutes in the ionic liquid 1-ethyl-3methylimidazolium trifluoromethanesulfonate using gas-liquid chromatography at $T=(313.15$, 323.15, and 333.15). J. Chem. Thermodyn., 42, 7883 (2010). https://doi.org/10.1016/j.jct.2009.07.010

Plechkova, N. V., Seddon, K. R. Applications of ionic liquids in the chemical industry. Chem. Soc. Rev., 37, 123-150 (2008). https://doi.org/10.1039/B006677J

Rogošić, M., Zagajski Kučan, K. Modeling of liquidliquid equilibria in quasi-seven-component systems with deep eutectic solvents as extraction media. Kem. Ind., 67 (9-10), 385-402 (2018). https://doi. org/10.15255/KUI.2018.004
Sander, A., Rogošić, M., Slivar, A., Žuteg, B. Separation of hydrocarbons by means of liquidliquid extraction with deep eutectic solvents. Solvent Extr. Ion Exc., 34, 86-98 (2016). https:// doi.org/10.1080/07366299.2015.1132060

Smith, L., Abbott, A. P., Ryder, K. S., Deep eutectic solvents (DESs) and their applications. Chem. Rev., 114, 11060-11082 (2014). https://doi.org/10.1021/ cr300162p

Sørensen, J. M., Arlt, W. Liquid-liquid equilibrium data collection, DECHEMA, Frankfurt (1979).

Verevkin, S. P., Sazonova, A. Y., Frolkova, A. K., Zaitsau, D. H., Prikhodko, I. V., Held, C., Separation performance of biorenewable deep eutectic solvents. Ind. Eng. Chem. Res., 54, 3498-3504 (2015). https://doi.org/10.1021/acs.iecr.5b00357

Zagajski Kučan, K., Perković, M., Cmrk, K. Načinović, D., Rogošić, M., Betaine + (glycerol or ethylene glycol or propylene glycol) deep eutectic solvents for extractive purification of gasoline. Chemistry Select, 3, 12582-12590 (2018). https:// doi.org/10.1002/slct.201803251

Zhang, S., Zhang, S., Zhang, Z. C. Extractive desulfurization and denitrogenation of fuels using ionic liquids. Ind. Eng. Chem. Res., 43, 614-622 (2004). https://doi.org/10.1021/ie030561+

Zhao, D., Liao, Y., Zhang, Z. Toxicity of ionic liquids, Water, 35, 42-48 (2007). https://doi.org/10.1002/ clen.200600015 